DOI: $10.24850 /$ j-tyca-2018-05-01

Artículos

\title{
Evolución temporal de las sequías hidrológicas en Argentina y su relación con indicadores macroclimáticos
}

\author{
Erica Díaz ${ }^{1}$ \\ Marcelo García ${ }^{2}$ \\ Andrés Rodríguez ${ }^{3}$ \\ Oscar Dölling ${ }^{4}$ \\ Santiago Ochoa ${ }^{5}$ \\ Juan Bertoni ${ }^{6}$ \\ ${ }^{1}$ Universidad Nacional de Córdoba, Córdoba, Argentina, \\ erica.betiana.diaz@unc.edu.ar \\ ${ }^{2}$ Universidad Nacional de Córdoba, Córdoba, Argentina, \\ carlos.marcelo.garcia@unc.edu.ar \\ ${ }^{3}$ Universidad Nacional de Córdoba, Córdoba, Argentina, \\ andres.rodriguez@unc.edu.ar \\ ${ }^{4}$ Universidad Nacional de San Juan, San Juan, Argentina, \\ odolling@unsj.edu.ar \\ ${ }^{5}$ Universidad Nacional de Córdoba, Córdoba, Argentina, \\ santiago.ochoa@unc.edu.ar \\ ${ }^{6}$ Universidad Nacional de Córdoba, Córdoba, Argentina, \\ jcbertoni@unc.edu.ar
}

Autora para correspondencia: Erica Díaz, erica.betiana.diaz@unc.edu.ar

\section{Resumen}

En este trabajo se identifican, a través de un análisis espectral, las periodicidades plurianuales en las series históricas de caudales medios anuales escurridos en 14 sistemas fluviales de la República Argentina y en 10 indicadores macroclimáticos. La identificación de las frecuencias de tiempo dominantes en las series de caudales escurridos permite comprender la escala temporal de evolución de los 
procesos físicos que intervienen en los ciclos hidrológicos. En el análisis realizado se encontró que existe en todas las series históricas de caudales medios anuales escurridos fluctuaciones de alta, baja y media frecuencia, que aportan, con distinta significancia, a la variabilidad temporal de los caudales escurridos. Los resultados se contrastaron con los obtenidos en el análisis de series de indicadores macroclimáticos, para saber si existía relación de la evolución de tales indicadores con las series de caudales. Los resultados alcanzados permiten avanzar en la generación de modelos conceptuales y de pronósticos implementados para prever los años de déficit y excesos hídricos en los sistemas fluviales, lo que constituye una herramienta importante para la planificación y gestión de los recursos hídricos.

Palabras clave: sequías hidrológicas, indicadores macroclimáticos.

\begin{abstract}
In this paper, multiannual periodicities in the historical time-series average annual streamflows discharges of 14 rivers of Argentina and in ten macroclimatic indicators are identified using spectral analysis. The estimation of dominant frequencies of the analyzed time series allows to understand the time scale of the processes involved in the hydrological cycles. The spectral analysis found that high, low and medium frequency fluctuations contribute, in different percentages, to the flow variability. Then, this results have been compared with results of similar analysis performed on time series of macro-climatic indicators to calculate the relation of the evolution of these indicators with streamflows discharge series. These results constitute an important step toward implementing conceptual and forecasting models of deficit and excess of surface water in fluvial system for planning and management of the water resources.
\end{abstract}

Keywords: Hydrological drought, macro-climatic indicators.

Recibido: $04 / 11 / 2016$

Aceptado: 03/04/2018

\title{
Introducción
}

Para una planificación y gestión adecuadas de los recursos hídricos se debe tener en cuenta la incertidumbre asociada con la variabilidad de 
la oferta y demanda de los recursos disponibles en una región. En ese aspecto, es clave conocer la evolución en tiempo y espacio de los recursos hídricos disponibles para la toma de decisiones e identificación de la fragilidad de los sistemas de una misma región.

Las situaciones de extremos hidrológicos, como periodos de excesos - escasez de agua, representan amenazas para la sociedad. En particular, las sequías, que son periodos de escasez, son fenómenos de relativa complejidad, que afectan de manera importante el desarrollo y aprovechamiento de los recursos hídricos en una región (Fernández, 1997). Avanzar en el conocimiento de sus características (frecuencia de ocurrencia, duración, extensión espacial, intensidad y magnitud) y su relación con los fenómenos macroclimáticos que intervienen en su formación, ayuda a la generación de modelos de predicción a mediano y largo plazos, que permitirían estar preparados para la gestión eficiente de los recursos hídricos durante los periodos de escasez.

En un trabajo previo realizado por los autores, se abordó la identificación y caracterización de las sequías en la República Argentina desde una concepción hidrológica (Díaz, Rodríguez, Dölling, Bertoni, \& Smrekar, 2016). En este trabajo se identifican las periodicidades plurianuales tanto de las series de caudales de los sistemas fluviales de la República Argentina como de indicadores macroclimáticos, utilizando un análisis espectral.

Conocer las frecuencias de tiempo dominantes (caracterizando las periodicidades plurianuales) en las series de caudales permite comprender la evolución temporal de los procesos que intervienen en los ciclos hidrológicos y así avanzar en la generación de modelos conceptuales que expliquen dichos procesos, al igual que desarrollar modelos de pronóstico que ayuden en distintas escalas a prever los años de déficit hídricos, así como en la planificación y gestión de los recursos hídricos.

\section{Región de estudio}

La región de estudio incluye 14 cuencas de las regiones Centro, Norte y Cuyo de la República Argentina. En la Tabla 1 y la Figura 1 se describen sus principales características. 
Tabla 1. Resumen de las características hidrológicas y geográficas de las cuencas analizadas.

\begin{tabular}{|c|c|c|c|c|c|c|c|c|}
\hline \multirow{3}{*}{$\begin{array}{l}\text { Identifica- } \\
\text { dor }\end{array}$} & \multirow{3}{*}{$\begin{array}{l}\text { Cuenca del } \\
\text { río }\end{array}$} & \multicolumn{4}{|c|}{ Estación de aforo } & \multirow{3}{*}{$\begin{array}{c}\text { Área de } \\
\text { Aporte } \\
\text { hasta la } \\
\text { estación } \\
\left(\mathrm{km}^{2}\right)\end{array}$} & \multirow{3}{*}{$\begin{array}{l}\text { Módulo } \\
\left(\mathrm{m}^{3} / \mathrm{s}\right)\end{array}$} & \multirow{3}{*}{$\begin{array}{l}\text { Periodo de } \\
\text { datos }\end{array}$} \\
\hline & & \multirow{2}{*}{ Denominación } & \multicolumn{3}{|c|}{ Localización } & & & \\
\hline & & & $\begin{array}{l}\text { Latitud } \\
\text { sur }\end{array}$ & $\begin{array}{l}\text { Longitud } \\
\text { oeste }\end{array}$ & $\begin{array}{l}\text { Altitud } \\
\text { (msnm) }\end{array}$ & & & \\
\hline 1 & San Juan & $\begin{array}{c}\text { San Juan - Km } \\
47.3\end{array}$ & $31^{\circ} 30^{\prime} 59^{\prime \prime}$ & $68^{\circ} 56^{\prime} 25^{\prime \prime}$ & 934 & 25660 & 65 & $1909-2013$ \\
\hline 2 & Mendoza & Guido & $32^{\circ} 54^{\prime} 55^{\prime \prime}$ & $69^{\circ} 14^{\prime} 16^{\prime \prime}$ & 1408 & 8180 & 45.6 & $1956-2013$ \\
\hline 3 & Atuel & La Angostura & $35^{\circ} 05^{\prime} 57^{\prime \prime}$ & $68^{\circ} 52^{\prime}$ & 6"1302 & 3800 & 35.4 & $1905-2013$ \\
\hline 4 & Colorado & Buta Ranquil & $37^{\circ} 04^{\prime} 34^{\prime \prime}$ & $69^{\circ} 44^{\prime} 48^{\prime \prime}$ & 850 & 15300 & 148 & $1940-2013$ \\
\hline 5 & $\begin{array}{l}\text { Ctalamo- } \\
\text { chita }\end{array}$ & Embalse & $32^{\circ} 10^{\prime} 00^{\prime \prime}$ & $64^{\circ} 23^{\prime} 00^{\prime \prime}$ & 650 & 3300 & 27.1 & $1913-1984$ \\
\hline 6 & Xanaes & Los Molinos & $31^{\circ} 48^{\prime} 10^{\prime \prime}$ & 6430'59" & 770 & 980 & 9.5 & $1936-2009$ \\
\hline 7 & Anizacate & Santa Ana & $31^{\circ} 40^{\prime} 00^{\prime \prime}$ & $64^{\circ} 34^{\prime} 00^{\prime \prime}$ & 900 & 465 & 4.83 & $1925-1980$ \\
\hline 8 & Suquía & San Roque & $31^{\circ} 22^{\prime} 00^{\prime \prime}$ & $64^{\circ} 27^{\prime} 00^{\prime \prime}$ & 650 & 1350 & 10 & $1926-2011$ \\
\hline 9 & Dulce & La Escuela & $\begin{array}{l}27^{\circ} 30^{\prime} \\
00^{\prime \prime}\end{array}$ & $\begin{array}{l}64^{\circ} 51^{\prime} \\
00^{\prime \prime}\end{array}$ & 265 & 19700 & 82.2 & $1925-2013$ \\
\hline 10 & Juramento & Cabra Corral & $25^{\circ} 16^{\prime} 19^{\prime \prime}$ & $65^{\circ} 19^{\prime} 47^{\prime \prime}$ & 945 & 32885 & 29.5 & $1934-2013$ \\
\hline 11 & Bermejo & Pozo Sarmiento & $23^{\circ} 13^{\prime} 00^{\prime \prime}$ & $64^{\circ} 12^{\prime} 00^{\prime \prime}$ & 296 & 25000 & 446 & $1952-2013$ \\
\hline 12 & Pilcomayo & La Paz & $22^{\circ} 22^{\prime} 41^{\prime \prime}$ & $62^{\circ} 31^{\prime} 21^{\prime \prime}$ & 230 & 96000 & 237 & $1941-2013$ \\
\hline 13 & Paraná & Corrientes & $27^{\circ} 28^{\prime} 30^{\prime \prime}$ & $58^{\circ} 49^{\prime} 60^{\prime \prime}$ & 52 & 1950000 & 17189 & $1906-2013$ \\
\hline 14 & Salado & $\begin{array}{c}\text { Ruta Provincial } \\
70\end{array}$ & $31^{\circ} 29^{\prime} 28^{\prime \prime}$ & $60^{\circ} 46^{\prime} 50^{\prime \prime}$ & 17 & 29700 & 137 & $1954-2013$ \\
\hline
\end{tabular}


Tecnología y
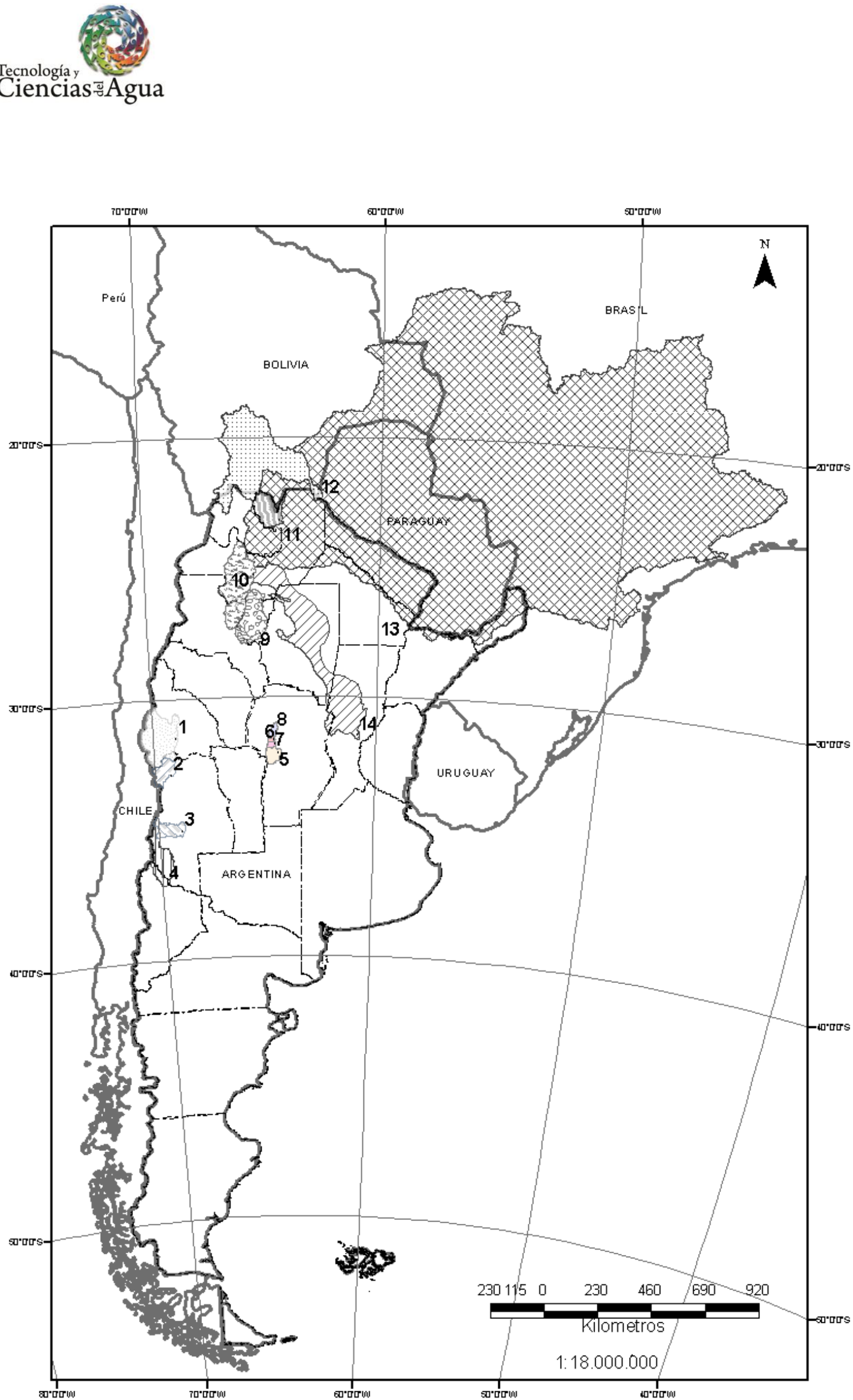
Figura 1. Ubicación de las cuencas analizadas en el trabajo. Los números corresponden con el indicador mostrado en la Tabla 1.

Las series de caudales analizadas corresponden a cuencas de la región Centro, Norte, Noa y Cuyo de la República Argentina mostradas en la Figura 1. Los ríos presentan características diferentes en cuanto a localización de la cuenca, módulo, área de aporte y volumen de aporte anual (ver Tabla 1).

La cuenca del río San Juan cubre gran parte de la provincia de San Juan y abarca un pequeño sector del norte de Mendoza. Casi la totalidad del escurrimiento superficial proviene del derretimiento de la nieve o hielo de los glaciares en los Andes, y constituye la principal fuente de recarga de los acuíferos.

La cuenca del río Mendoza se ubica en el extremo noroeste de la provincia homónima y cubre una pequeña extensión del sur de la provincia de San Juan. Esta cuenca drena $90 \mathrm{~km}$ del frente de la cordillera de los Andes. Las aguas de este río provienen en su mayoría del deshielo. El río Atuel es el quinto afluente del desaguadero; es de régimen nival, aunque también recibe aportes pluviales, y tiene una longitud aproximada de $600 \mathrm{~km}$. Su cuenca de alimentación está a más de 3000 msnm en la cordillera de los Andes.

La cuenca del río Colorado (Subsecretaría de Recursos Hídricos, 2010) comprende cuatro ecorregiones con relieve y régimen de precipitaciones muy variado, desde 100 hasta $600 \mathrm{~mm}$ medios anuales. Es una cuenca pluvionival.

La cuenca del río Ctalamochita pertenece a la cuenca Carcaraña y es de carácter exorreico, terminando en la cuenca del Plata. Nace en las vertientes orientales de la Sierra Grande. En la actualidad, el río Tercero (Ctalamochita) está regulado por una cadena de reservorios artificiales.

El río Xanaes nace por la confluencia de los ríos Anisacate y de Los Molinos. El río Anisacate resulta de la unión de los ríos de la Suela y el San José. Atraviesa la Sierra Chica en una garganta estrecha como río retrogradante. El río Suquía posee una cuenca de unos $6000 \mathrm{~km}^{2}$ cerca de Mar Chiquita y adquiere su nombre en el embalse San Roque. En el punto de estudio, el área de la cuenca es de $1350 \mathrm{~km}^{2}$. Estos últimos son típicos ríos de montaña, donde las variaciones de caudal responden directamente a las variaciones de las precipitaciones en la cuenca. Los caudales por arriba del módulo suceden entre octubre y marzo; mientras que los estiajes son más acentuados y abarcan el periodo junio-septiembre. 
La cuenca de aporte del río Dulce en el tramo estudiado abarca las provincias de Tucumán, Salta y Catamarca. Presenta una precipitación promedio anual de $800 \mathrm{~mm}$ y se considera de clima húmedo.

La cuenca del río Pasaje-Juramento tiene sus nacientes en las cumbres de los nevados de Cachi y Acay (4 $895 \mathrm{msnm}$ ), en la cordillera oriental. La cuenca alta y media se desarrolla dentro de la provincia de Salta. El río Juramento está regulado a través del dique Embalse General Belgrano (Cabra Corral), de propósito múltiple. La cuenca del río Bermejo forma parte de la hoya hidrográfica del Plata y junto con el río Pilcomayo es el principal afluente del río Paraguay. La cuenca alta del río Bermejo se sitúa en el extremo noroeste de Argentina y extremo sursudeste de Bolivia. La precipitación anual media varía desde $200 \mathrm{~mm}$ en la parte oeste hasta $1400 \mathrm{~mm}$ en el centro de la cuenca. El Pilcomayo es un río de montaña que tiene sus nacientes en los Andes bolivianos, a más de $5000 \mathrm{~m}$ de alturas snm; pasa en dirección NO-SE por las sierras subandinas.

A lo largo de los tiempos geológicos, el río fue depositando gran parte de los sedimentos que transporta sobre la planicie chaqueña, construyendo así un gran abanico aluvial. La cuenca del río Paraná en el punto de análisis (estación Corrientes) tiene un área de 1950000 $\mathrm{km}^{2}$. Esta cuenca es parte de la cuenca del Plata, una de los mayores del mundo. Se nota una escasez pluvial en invierno (junio-agosto) en la mayor parte de la cuenca, con lluvias intensas en verano, siendo más reducidas en el oeste y en las regiones situadas al norte del trópico de capricornio, hasta el Planalto de Brasil; hacia el sur se caracteriza por abundantes precipitaciones. La precipitación media anual es de 1200 mm (1961-1990) (Paoli \& Schreider, 2000). El río Salado pertenece al complejo hídrico de la cuenca del Plata. Las fuentes del río Salado surgen en las estribaciones orientales de los Andes dentro de la provincia de Salta; su fuente principal se encuentra en la llamada Sierra de los Pasos Grandes, casi inmediatamente al sur del cerro nevado de Acay. Es un río de régimen pluvionival.

\section{Metodología y resultados}

\section{Análisis espectral en las series de caudales medios anuales}


El análisis espectral consistió en calcular, en primera instancia, los espectros de energía de las series históricas de caudales medios anuales, con el objetivo de identificar las frecuencias dominantes. En el análisis espectral se analiza la serie de caudales en el dominio de la frecuencia, utilizando la transformada rápida de Fourier. Después, el espectro de energía de la fluctuación se calculó con la siguiente ecuación:

$E=\frac{2 \Delta t}{N}\left\lfloor X_{m}\right\rfloor^{2}$

Donde:

$\Delta t$ : paso de tiempo utilizado igual a un año para este estudio.

$N$ : números de datos en la serie.

$\left[X_{m}\right\rfloor^{2}$ : magnitud de la transformada de Fourier.

A continuación (Figura 2, Figura 3, Figura 4, Figura 5, Figura 6, Figura 7, Figura 8, Figura 9, Figura 10, Figura 11, Figura 12, Figura 13, Figura 14 y Figura 15) se presentan las series de caudales medios anuales con sus respectivos espectros adimensionales de energía de las fluctuaciones de los caudales (utilizando el valor máximo para su adimensionalización), resaltando las tres frecuencias consideradas dominantes (máximo valor) en cada espectro (Tabla 2).
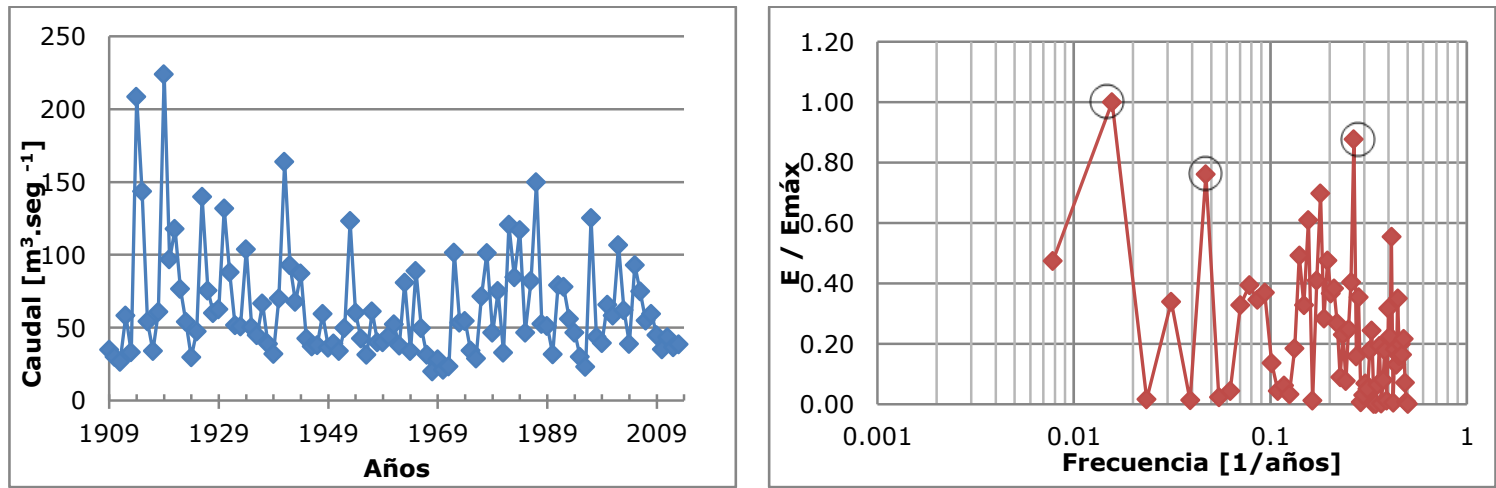

Figura 2. Serie de caudales del río San Juan y espectro de energía de las fluctuaciones de los caudales. 

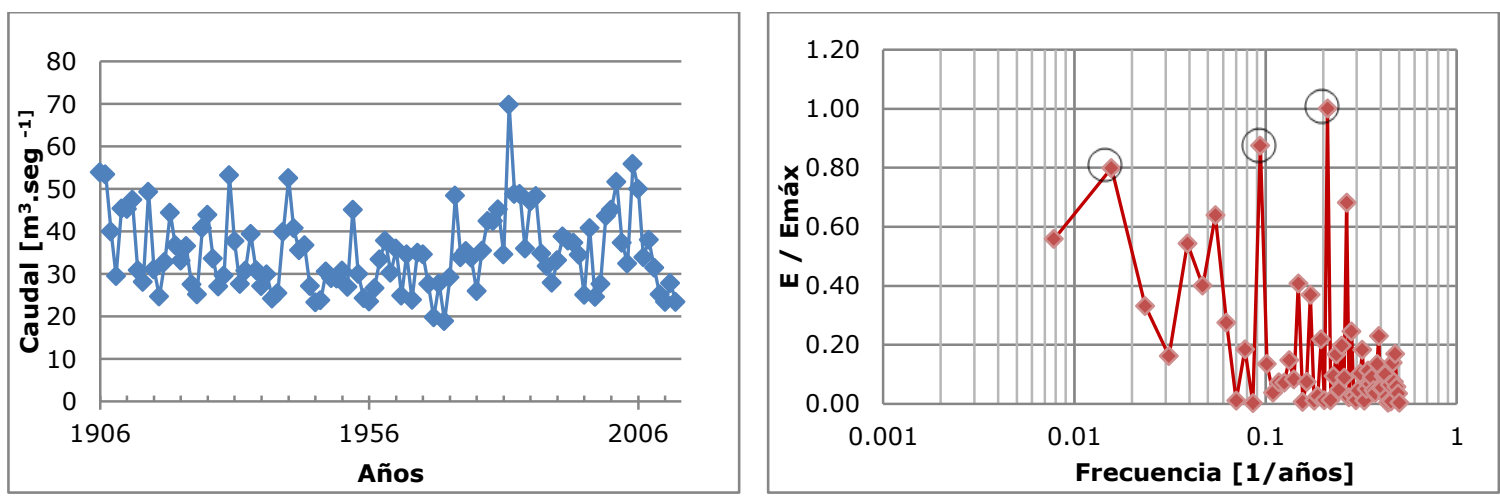

Figura 3. Serie de caudales del río Atuel y espectro de energía de las fluctuaciones de los caudales.
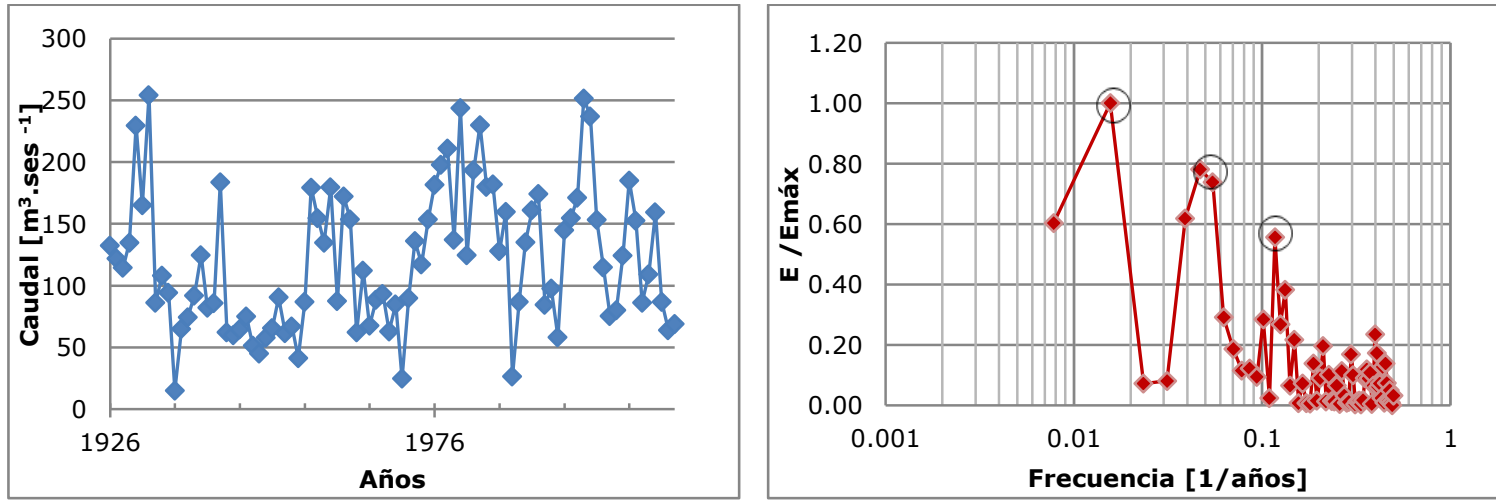

Figura 4. Serie de caudales del río Dulce y espectro de energía de las fluctuaciones de los caudales.
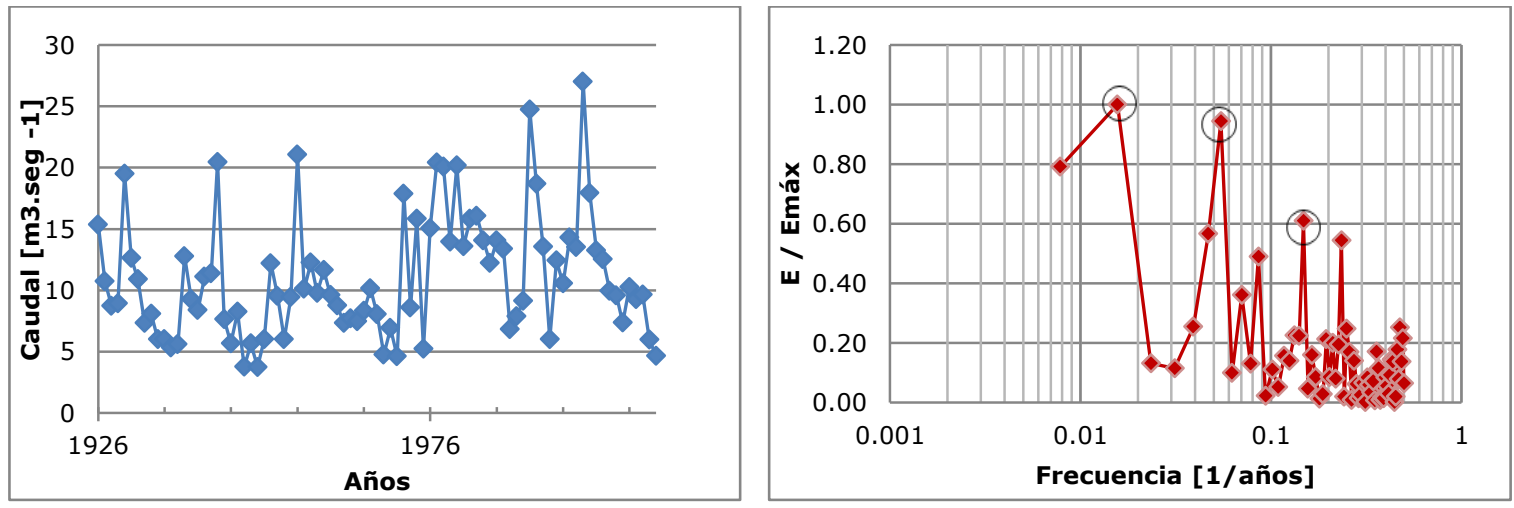

Figura 5. Serie de caudales del río Suquía y espectro de energía de las fluctuaciones de los caudales. 

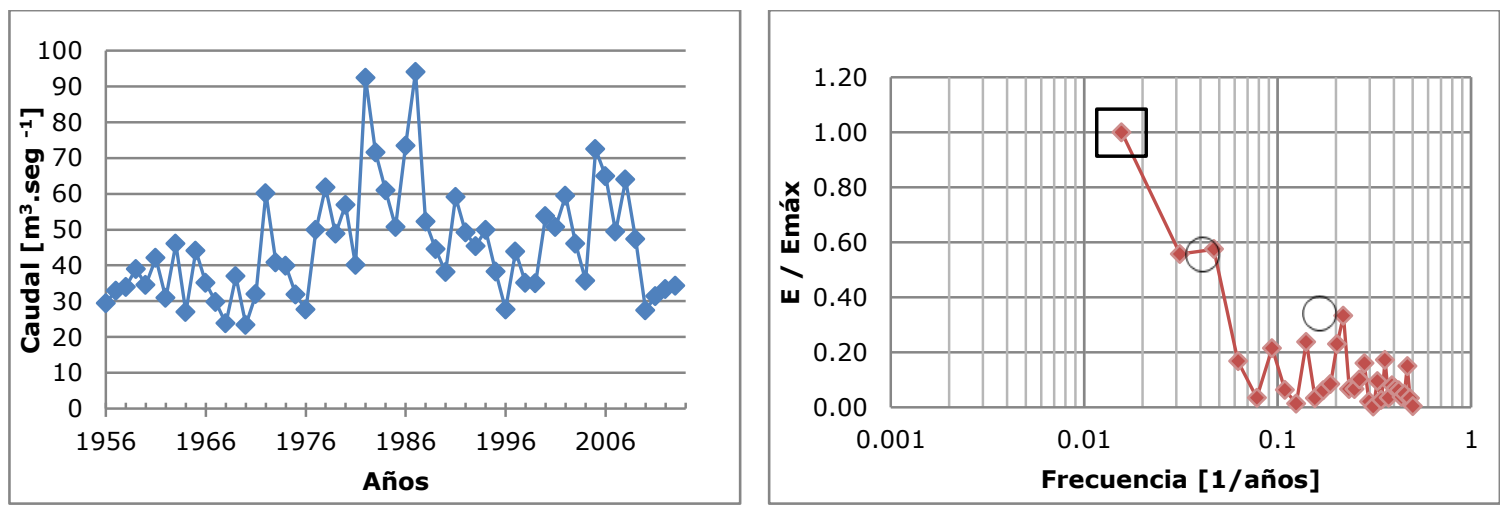

Figura 6. Serie de caudales del río Mendoza y espectro de energía de las fluctuaciones de los caudales.
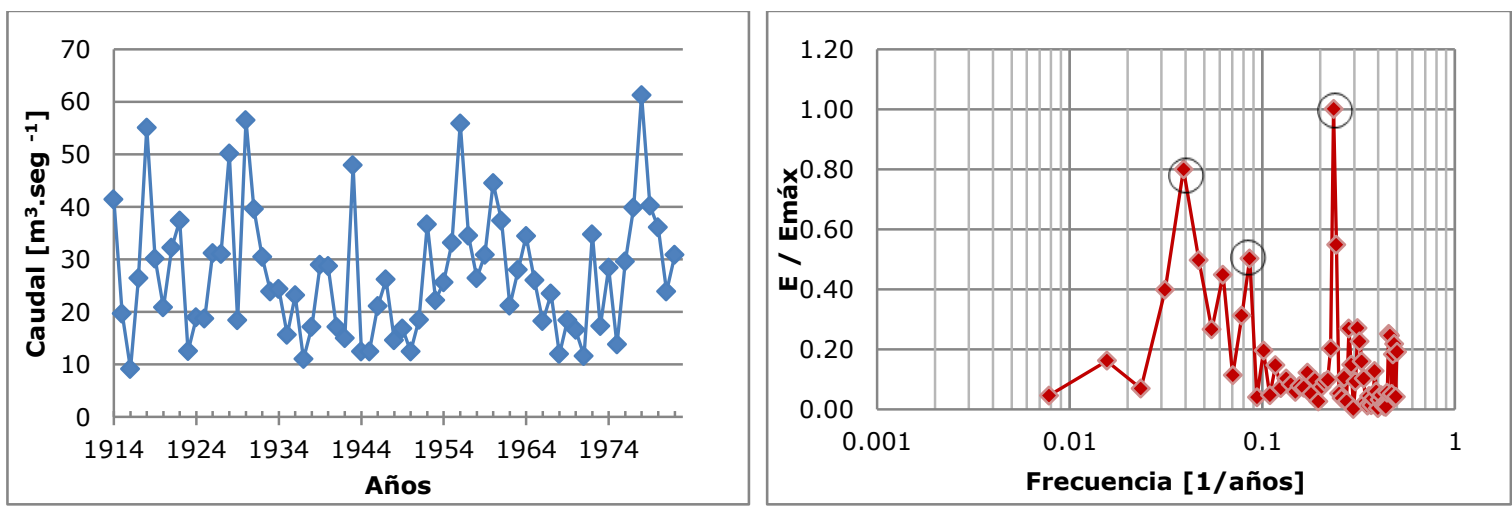

Figura 7. Serie de caudales del río Ctalanochita y espectro de energía de las fluctuaciones de los caudales.
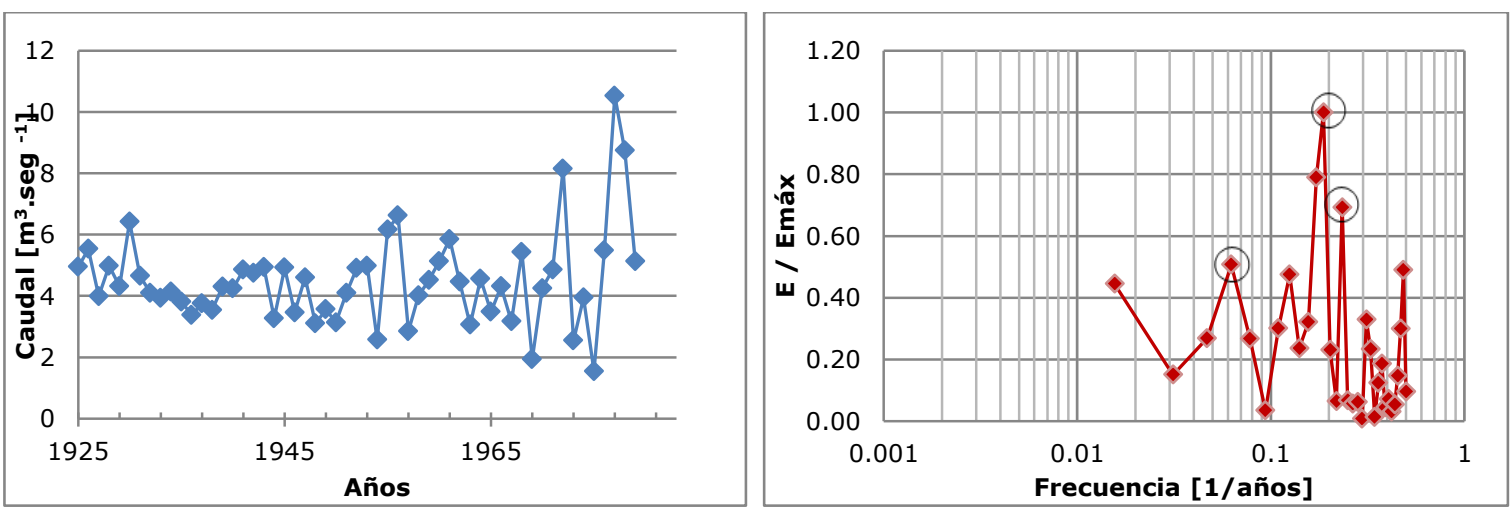

Figura 8. Serie de caudales del río Anizacate y espectro de energía de las fluctuaciones de los caudales. 

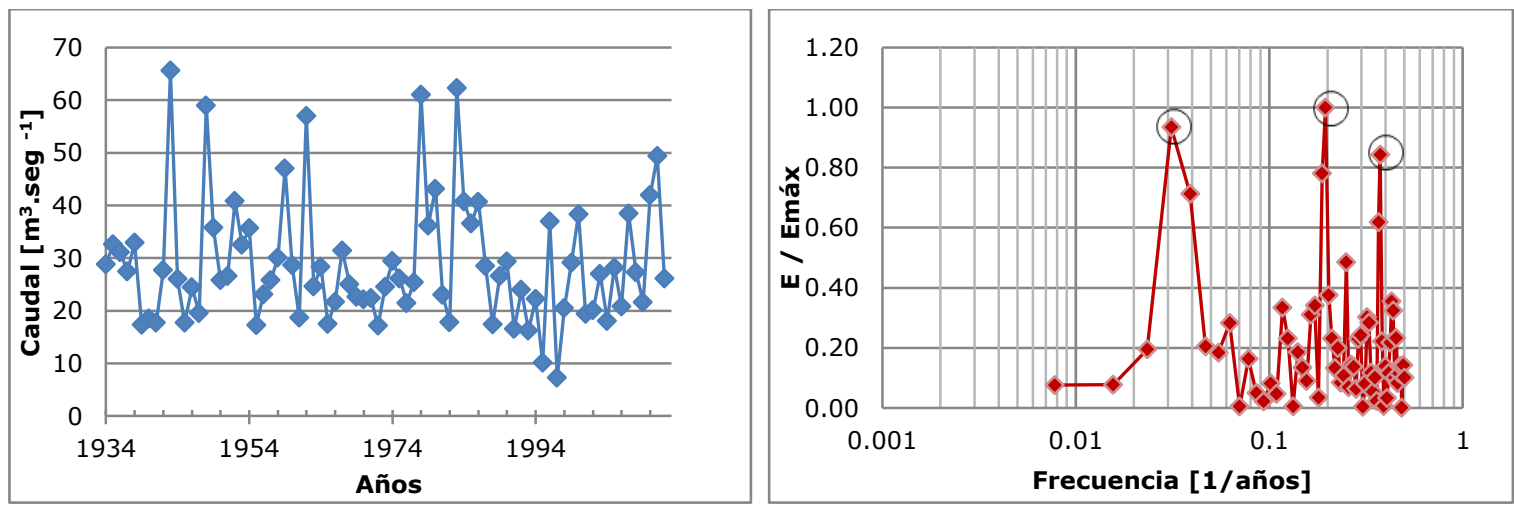

Figura 9. Serie de caudales del río Juramento y espectro de energía de las fluctuaciones de los caudales.
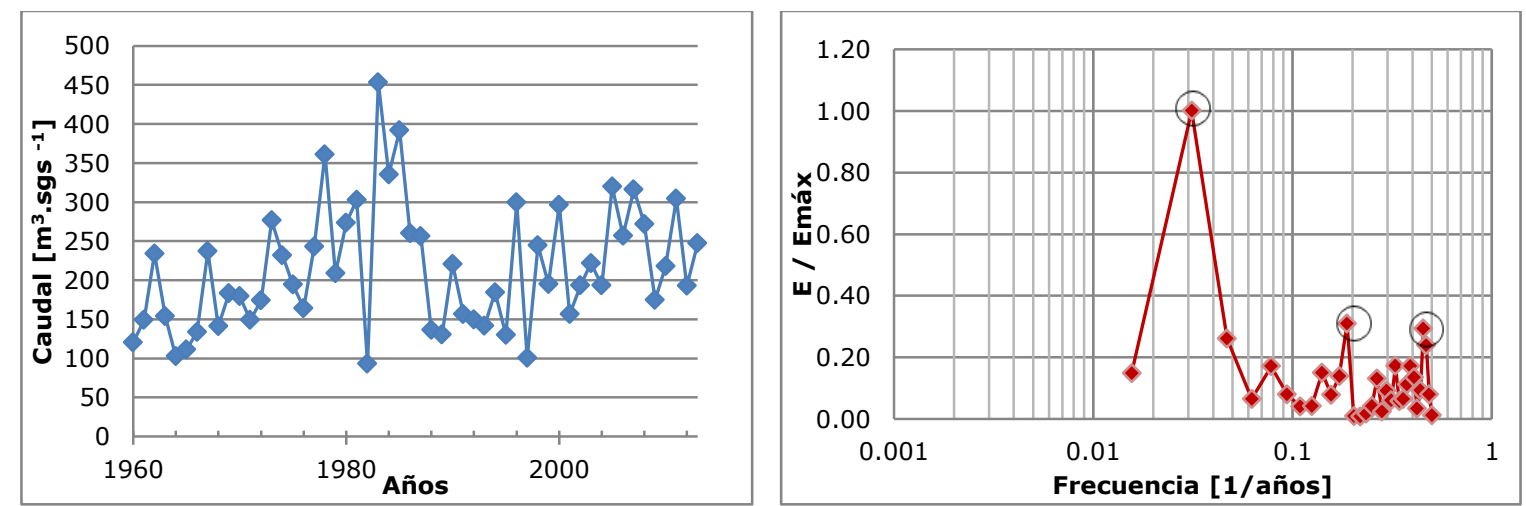

Figura 10. Serie de caudales del río Pilcomayo y espectro de energía de las fluctuaciones de los caudales.
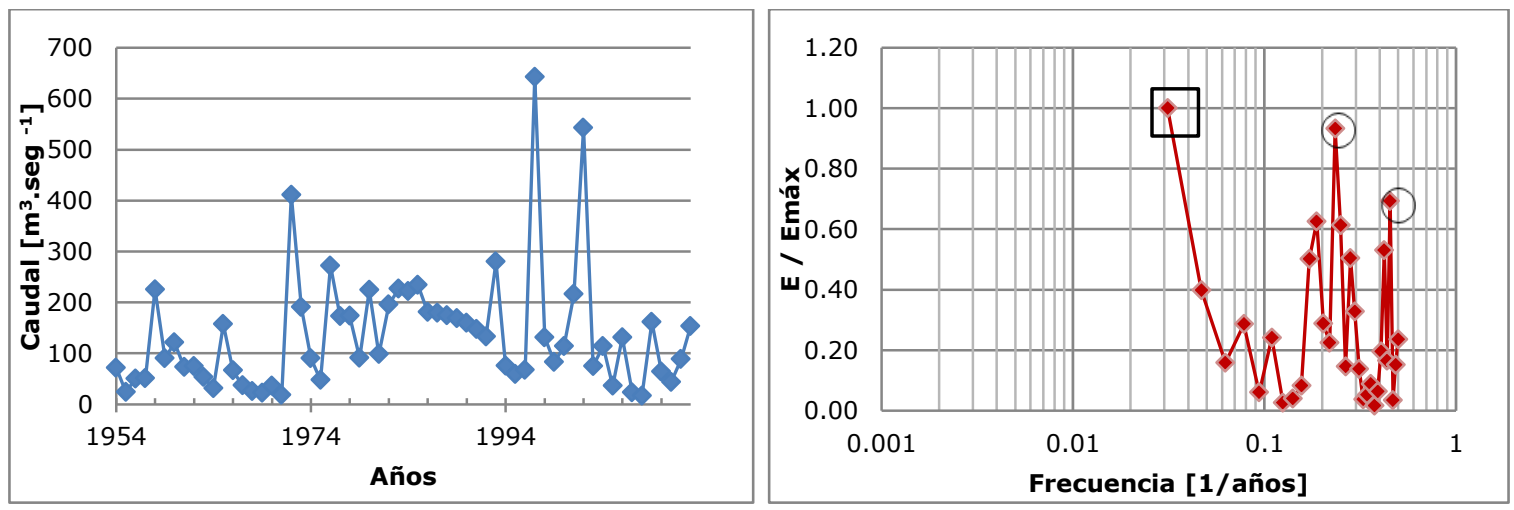

Figura 11. Serie de caudales del río Salado y espectro de energía de las fluctuaciones de los caudales. 

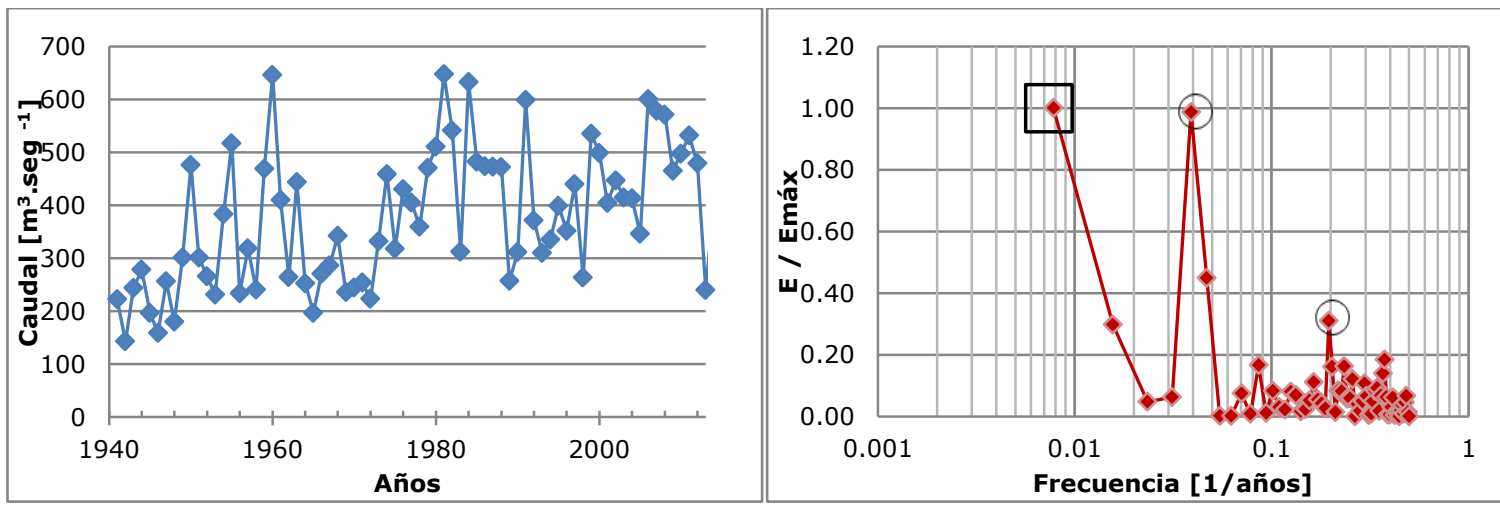

Figura 12. Serie de caudales del río Bermejo y espectro de energía de las fluctuaciones de los caudales.
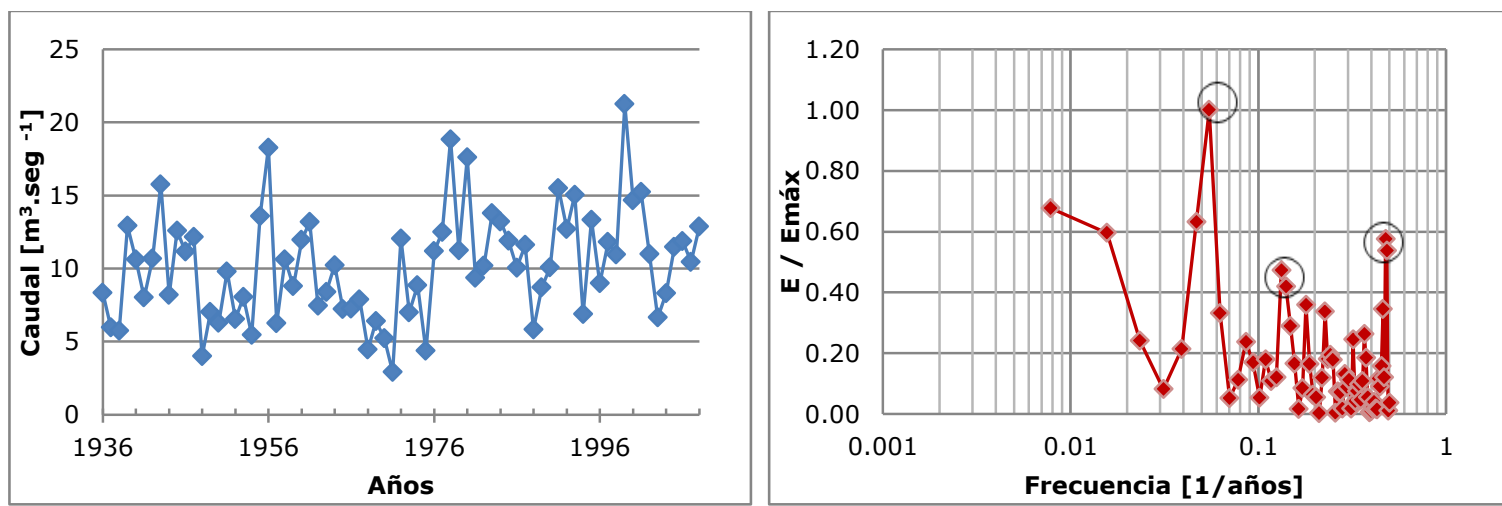

Figura 13. Serie de caudales del río Xanaes y espectro de energía de las fluctuaciones de los caudales.
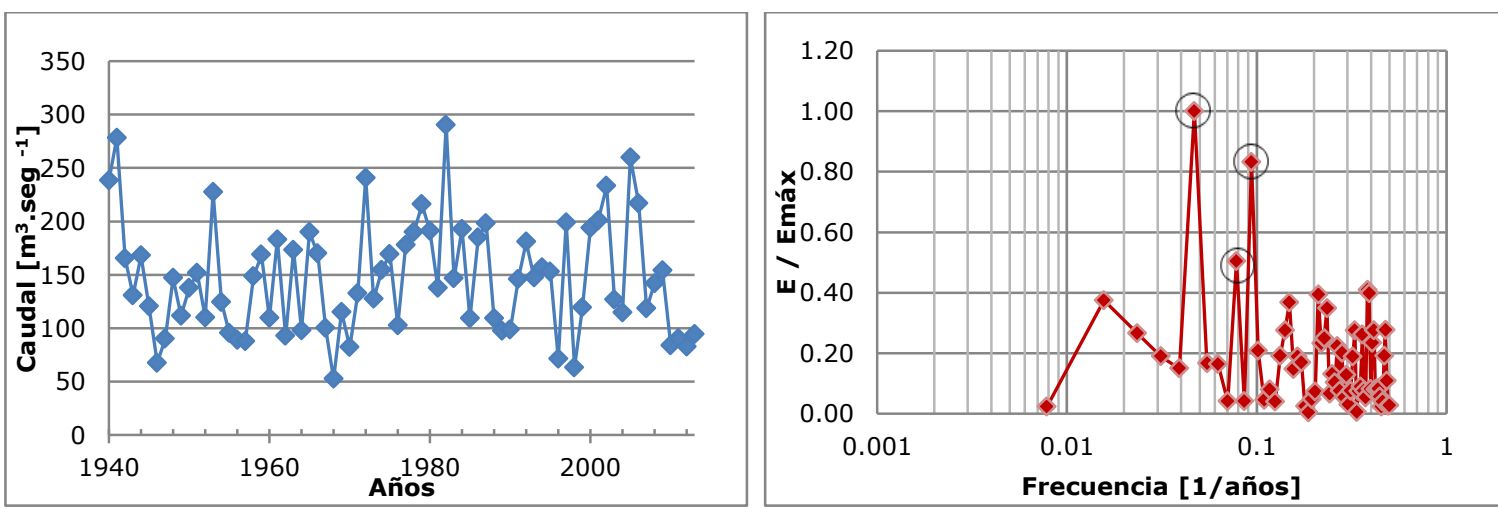

Figura 14. Serie de caudales del río Colorado y espectro de energía de las fluctuaciones de los caudales. 

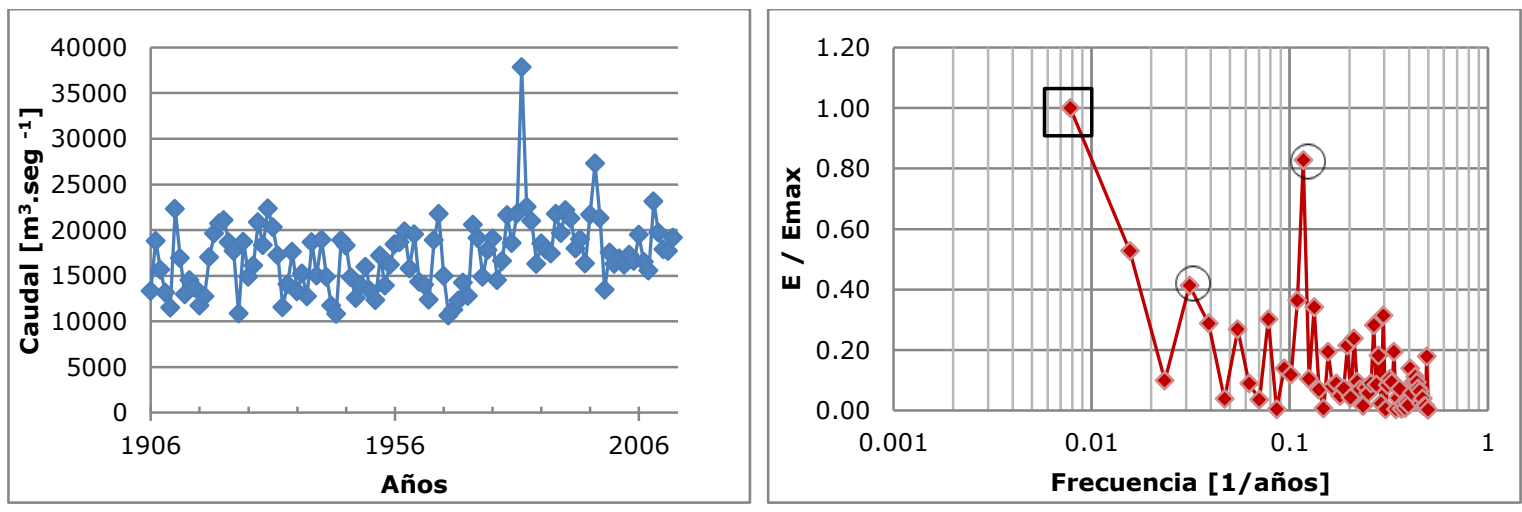

Figura 15. Serie de caudales del río Paraná y espectro de energía de las fluctuaciones de los caudales.

Tabla 2. Frecuencias dominantes y energía del espectro (adimensionalizada con su valor máximo) de cada cuenca estudiada.

\begin{tabular}{|c|c|c|c|c|c|c|}
\hline \multirow{2}{*}{ Cuencas } & \multicolumn{3}{|c|}{ Valor máximo seleccionado } & \multicolumn{3}{c|}{ Segundo valor seleccionado } \\
\cline { 2 - 7 } & E/Emáx & Frecuencia (1/año) & Periodo (años) & E/Emáx & Frecuencia (1/año) & Periodo (años) \\
\hline San Juan & 0.88 & 0.27 & 3.8 & 0.76 & 0.05 & 21.3 \\
\hline Mendoza & 0.33 & 0.22 & 21.3 & 0.23 & 0.20 & 4.6 \\
\hline Atuel & 1.00 & 0.21 & 4.7 & 0.88 & 0.09 & 10.7 \\
\hline Colorado & 1.00 & 0.05 & 21.3 & 0.83 & 0.09 & 10.7 \\
\hline Ctalamochita & 1.00 & 0.23 & 4.3 & 0.80 & 0.04 & 25.6 \\
\hline Xanaes & 1.00 & 0.05 & 18.3 & 0.58 & 0.48 & 2.1 \\
\hline Anizacate & 1.00 & 0.19 & 5.3 & 0.69 & 0.23 & 16 \\
\hline Suquía & 0.94 & 0.05 & 18.3 & 0.61 & 0.15 & 6.7 \\
\hline Dulce & 0.78 & 0.05 & 21.3 & 0.56 & 0.12 & 8.5 \\
\hline Juramento & 1.00 & 0.20 & 5.1 & 0.93 & 0.03 & 32 \\
\hline Bermejo & 0.99 & 0.04 & 25.6 & 0.31 & 0.20 & 5.1 \\
\hline Pilcomayo & 0.31 & 0.19 & 5.33 & 0.29 & 0.45 & 2.21 \\
\hline Paraná & 0.83 & 0.12 & 8.5 & 0.41 & 0.03 & 32 \\
\hline Salado & 0.93 & 0.22 & 4.6 & 0.69 & 0.44 & 2.3 \\
\hline
\end{tabular}

En las figuras $6,11,12$ y 15 se marcó con un rectángulo la primera frecuencia dominante del espectro. Estas frecuencias (mayores al largo de la serie) no se tuvieron en cuenta porque con el análisis de series sintéticas se demostró que son producto de tendencias en la serie o componentes de la baja frecuencia del largo de la serie.

Se puede observar en la Tabla 3 que los dos periodos dominantes se dan en los siguientes rangos: 
$<3.7$ años

$3.7-6.7$ años

$8.5-10.7$ años

10.7 - 18.3 años

$18.3-32$ años
2 casos

9 casos

4 casos

2 casos

11 casos

Tabla 3. Frecuencias dominantes de algunas de las cuencas estudiadas.

\begin{tabular}{|c|c|c|c|c|}
\hline \multirow{2}{*}{ Cuencas } & \multicolumn{2}{|c|}{ Fourier } & \multicolumn{2}{c|}{ Wavelet } \\
\cline { 2 - 5 } & $\begin{array}{c}\text { Periodo } \\
\text { (años) }\end{array}$ & $\begin{array}{c}\text { Periodo } \\
\text { (años) }\end{array}$ & $\begin{array}{c}\text { Periodo } \\
\text { (años) }\end{array}$ & $\begin{array}{c}\text { Periodo } \\
\text { (años) }\end{array}$ \\
\hline Atuel & 4.7 & 10.7 & 4.1 & 23.4 \\
\hline Ctalamochita & 4.3 & 25.6 & 4.1 & 23.4 \\
\hline Anizacate & 5.3 & 16 & 5.8 & 16.5 \\
\hline Suquía & 18.3 & 6.7 & 16.5 & 4.1 \\
\hline Dulce & 21.3 & 8.5 & 8.3 & 23.4 \\
\hline Paraná & 8.5 & 32 & 8.3 & 33.1 \\
\hline
\end{tabular}

Se observa que para la mayoría de los ríos (excepto Colorado, Paraná y Dulce) se presentan fluctuaciones con periodos dominante entre 3 y 7 años. Periodos dominantes entre 7 y 11 años se observan en los ríos Colorado, Paraná, Atuel y Dulce; mientras que periodos dominantes entre 13 y 35 años se ve en todos los ríos, excepto Atuel, Salado y Pilcomayo. Existen resultados similares en trabajos de otros autores, como Vargas, Minetti y Poblete (2002), quienes detectaron fluctuaciones de baja frecuencia cuasi periódicas (de 22 a 26 años) en los caudales anuales del río Paraná; Compagnucci, Berman, Velasco-Herrera y Silvestri (2014) encontraron en la serie de caudales del río Paraná periodicidades de 3.5, 9 y 30 años, y en la serie de caudales del río Atuel, periodicidades de 4 a 5 años, 7,11 y 22 años.

En la serie de caudales del río San Juan se apreciaron cinco bandas de frecuencia con amplitudes significativas, que se corresponden con periodos de unos 12.3, 7.4, 5.7 y 3.8 años (Correa \& Guevara, 1992). 
Además de efectuar el análisis espectral con Fourier, se realizó un análisis de coherencia Wavelet en las series de caudales para validar los resultados obtenidos. Para las señales que presentan múltiples escalas de variabilidad y frecuencia en el espacio o tiempo puede ser de utilidad un análisis localizado de transformaciones con curvas tipo wavelets, a fin de discriminar las geometrías dominantes de las variables analizadas. La coincidencia que muestran los resultados de frecuencias dominantes obtenidos con las metodologías de análisis espectral con base en la transformada de Fourier y curvas tipo Wavelets son una muestra de que las señales analizadas no tienen un alto grado de variabilidad de frecuencias dominantes y, por lo tanto, con la aplicación del análisis espectral con base en Fourier se alcanzarían resultados satisfactorios.

Se realizó el análisis con la función Wavelet tipo Morlet $(k o=6)$.

\section{Análisis de la contribución de distintos procesos a las fluctuaciones de las series de caudales}

Después de realizar el análisis de frecuencias dominantes de las distintas series de caudales y encontrar que existen rangos de frecuencias que predominan en las series, se procedió a realizar un segundo análisis, que es el cálculo de la varianza. Dicho cálculo se llevó a cabo integrando el espectro de energía de las fluctuaciones en el rango de frecuencias analizado (Figura 16). Esto se realiza con el fin de evaluar el aporte porcentual que tienen a la varianza total y las varianzas de las fluctuaciones de cada rango de frecuencia característicos de los distintos procesos. Para efectuar esto, se procedió a aplicar filtros pasa-banda a los espectros entre los siguientes rangos de frecuencia: 


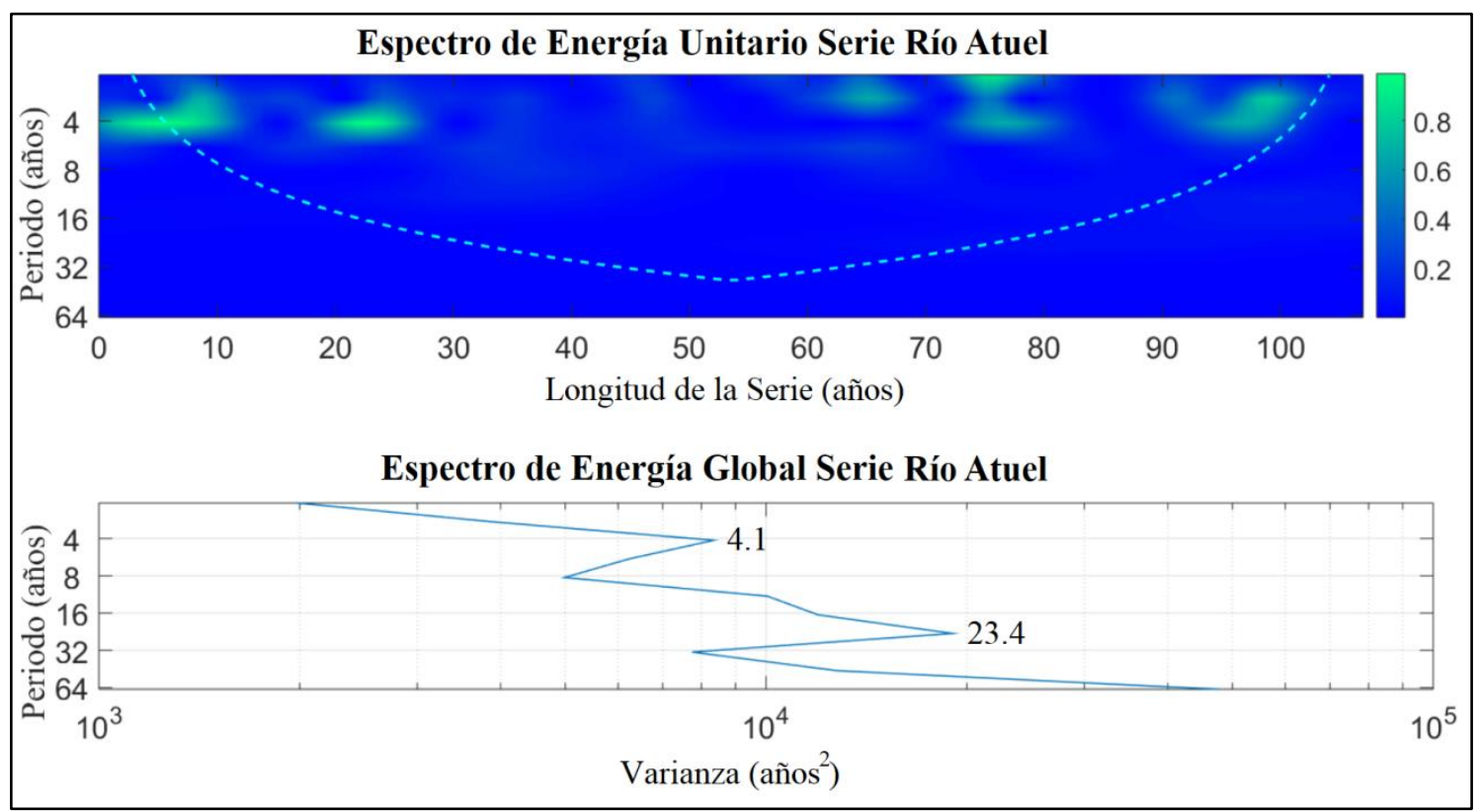

Figura 16. Espectro de energía unitario y global de las fluctuaciones de los caudales del río Atuel.

$N_{a}$ : con periodos de las fluctuaciones menores a 2.2 años y representa la variabilidad aleatoria.

$N_{2}$ : con periodos de las fluctuaciones entre 2.2 años y 3 años.

$N_{5}$ : con periodos de las fluctuaciones entre 3 y 7 años.

$N_{11}$ : con periodos de las fluctuaciones entre 7 y 13 años.

$N_{b d}$ : con periodos de las fluctuaciones entre 13 y 35 años.

$N_{v}$ : con periodos de las fluctuaciones mayores a 35 años.

El filtro pasa banda utilizado consiste en un filtro de Fourier (en primer lugar se verificó la herramienta con el análisis de una serie sintética, fluctuaciones de periodos conocidos), haciendo que la energía de las fluctuaciones sean iguales a cero para los rangos de frecuencias no incluidos en la banda del filtro pasa-banda utilizado. La Tabla 4 resume los resultados, donde se indica el porcentaje de aporte y la varianza que tienen las fluctuaciones de cada rango de frecuencia.

La suma distinta al $100 \%$ se debe a la correlación entre las componentes. 
En $N_{a}$ se consideran los procesos menores a 2.2 años y la parte aleatoria de todas las frecuencias. Correa y Guevara (1992) proponen que la varianza residual no explicada por las periodicidades significativas debe ser atribuida a la componente estocástica. Esta varianza aleatoria para $86 \%$ de los casos representa entre 30 y $50 \%$ de la varianza de las fluctuaciones, lo cual significa que la serie tiene un alto componente aleatorio. El resto puede explicarse en parte por distintos procesos con frecuencias características entre 2 y 3 años, 3 y 7 años, 7 y 13 años, 13 y 35 años y mayores a 35 años.

Se resalta en la Tabla 4 ( $\sin$ considerar los procesos $N_{a}$ ) que para nueve cuencas de 14 estudiadas, los procesos de baja frecuencia dominan $N_{b d}$ y luego lo siguen en importancia los $N_{5}$. Para dos cuencas se resalta el proceso $N_{v}$ y para sólo una, el proceso $N_{11}$ (Paraná). Así, el análisis reveló que existe en las series históricas de caudales medios anuales escurridos fluctuaciones de alta, baja y media frecuencia, que aportan, con distinta significancia, a la variabilidad temporal de los caudales escurridos. Este resultado permite avanzar en el estudio de los procesos que se deben considerar en la explicación de fenómenos hidrológicos, como sequías y excesos hídricos, teniendo en cuenta que el proceso involucra fenómenos que tienen en el tiempo una evolución que incluye procesos a pequeña, media y gran escala, y aleatorios o estocásticos.

Tabla 4. Aporte a la varianza total de las fluctuaciones de las series de caudales en cada ancho de banda.

\begin{tabular}{|c|c|c|c|c|c|c|c|}
\hline \multirow{2}{*}{ Cuenca } & \multicolumn{6}{|c|}{$\begin{array}{c}\text { Varianza total de las fluctuaciones de las } \\
\text { series de caudales en cada ancho de banda }\end{array}$} \\
\cline { 2 - 8 } & $\boldsymbol{N}_{\mathbf{a}}$ & $\mathbf{N}_{\mathbf{2}}$ & $\mathbf{N}_{\mathbf{5}}$ & $\mathbf{N}_{\mathbf{1 1}}$ & $\mathbf{N}_{\boldsymbol{b d}}$ & $\mathbf{N}_{\mathbf{v c}}$ & Suma \\
\hline San Juan & $45 \%$ & $3 \%$ & $27 \%$ & $5 \%$ & $4 \%$ & $7 \%$ & 87 \\
\hline Mendoza & $40 \%$ & $0 \%$ & $9 \%$ & $3 \%$ & $20 \%$ & $3 \%$ & 75 \\
\hline Atuel & $36 \%$ & $2 \%$ & $23 \%$ & $9 \%$ & $15 \%$ & $11 \%$ & 96 \\
\hline Colorado & $50 \%$ & $8 \%$ & $12 \%$ & $11 \%$ & $10 \%$ & $1 \%$ & 92 \\
\hline Ctalamochita & $50 \%$ & $0 \%$ & $19 \%$ & $8 \%$ & $19 \%$ & $0 \%$ & 96 \\
\hline Xanaes & $43 \%$ & $0 \%$ & $9 \%$ & $8 \%$ & $15 \%$ & $9 \%$ & 84 \\
\hline Anizacate & $19 \%$ & $2 \%$ & $38 \%$ & $12 \%$ & $5 \%$ & $0 \%$ & 76 \\
\hline Suquía & $38 \%$ & $0 \%$ & $14 \%$ & $8 \%$ & $15 \%$ & $15 \%$ & 90 \\
\hline Dulce & $28 \%$ & $6 \%$ & $4 \%$ & $16 \%$ & $25 \%$ & $16 \%$ & 95 \\
\hline Juramento & $49 \%$ & $12 \%$ & $22 \%$ & $1 \%$ & $12 \%$ & $0 \%$ & 96 \\
\hline Bermejo & $27 \%$ & $4 \%$ & $19 \%$ & $3 \%$ & $23 \%$ & $20 \%$ & 96 \\
\hline Pilcomayo & $33 \%$ & $6 \%$ & $9 \%$ & $4 \%$ & $26 \%$ & $0 \%$ & 78 \\
\hline
\end{tabular}




\begin{tabular}{|c|c|c|c|c|c|c|c|}
\hline Paraná & $31 \%$ & $3 \%$ & $16 \%$ & $21 \%$ & $9 \%$ & $16 \%$ & 96 \\
\hline Salado & $30 \%$ & $5 \%$ & $36 \%$ & $2 \%$ & $2 \%$ & $2 \%$ & 77 \\
\hline
\end{tabular}

\section{Relaciones entre la evolución temporal de sequías hidrológicas e indicadores macroclimáticos}

En esta sección se analizan indicadores macroclimáticos y astronómicos, utilizando el mismo análisis espectral de las series temporales. Para conocer si existe una relación entre su evolución temporal con las sequías hidrológicas en las distintas escalas de tiempo.

En la Tabla 5 se describen los indicadores macroclimáticos analizados.

Tabla 5. Resumen de los indicadores macroclimáticos utilizados.

\begin{tabular}{|c|c|c|}
\hline Indicador & Descripción & Periodo \\
\hline TNA & Índice del Atlántico norte tropical & $1948-2013$ \\
\hline TSA & Índice del Atlántico sur tropical & $1948-2013$ \\
\hline AMO & Oscilación multidecadal del Atlántico & $1861-2008$ \\
\hline AMM & Modo meridional del Atlántico & $1948-2001$ \\
\hline SOI & Índice de oscilación sur & $1951-2013$ \\
\hline PDO & Oscilación decadal del Pacífico & $1948-2013$ \\
\hline MS & Índice de El Niño oceánico & $1700-2013$ \\
\hline ONI & las regiones El Niño 3 y El Niño 4 & $1950-2012$ \\
\hline Niño 3.4 & $\begin{array}{c}\text { Temperatura promedares } \\
\text { Niño 1+2 }\end{array}$ & $\begin{array}{c}\text { Temperatura promedio de la superficie del mar en } \\
\text { las regiones 2+1 }\end{array}$ \\
\hline
\end{tabular}

Los indicadores seleccionados son ONI, AMM, TSA, AMO, SOI, PDO, Niño $1+2$, Niño 3.4 y TNA (ver Tabla 5). En la Tabla 5 y Tabla 6 se indican los periodos dominantes observados de las fluctuaciones y los aportes a la varianza (en porcentaje) de las fluctuaciones con los distintos anchos de bandas analizados anteriormente.

Tabla 6. Periodos dominantes en cada serie de indicadores analizados. 


\begin{tabular}{|c|c|c|c|c|c|c|c|c|c|c|c|}
\hline \multicolumn{2}{|r|}{ Indicadores } & MS & AMO & AMM & ONI & PDO & SOI & Niño & Niño & TSA & TNA \\
\hline \multirow{2}{*}{$\begin{array}{l}\frac{0}{0} \\
\text { 은 } \\
\text { ¿ }\end{array}$} & $\begin{array}{l}10 \text { valor máximo } \\
\text { seleccionado (años) }\end{array}$ & 10.9 & 64.0 & 9.1 & 4.9 & 21.0 & 12.8 & 4.9 & 4.9 & 11.6 & 8.5 \\
\hline & $\begin{array}{l}2^{\circ} \text { valor máximo } \\
\text { seleccionado (años) }\end{array}$ & 10.0 & 32.0 & 2.9 & 3.6 & 5.6 & 4.9 & 3.5 & 3.5 & 21.3 & 21.5 \\
\hline
\end{tabular}

Las series de indicadores AMO, AMM, ONI, PDO, SOI, Niño 3.4, Niño $1+2$, TSA y TNA se obtuvieron del sitio web del NOAA (2016), mientras que la serie de manchas solares se obtuvo de SILSO (2016).

En los índices PDO, TNA, AMM se encuentra un periodo de frecuencia dominante de 64 años $^{-1}$ y en el índice TSA de 128 años $^{-1}$; sin embargo, se descartan por presentar mucha incertidumbre, pues las series muestran longitudes inferiores a cien años. De este análisis es posible ver que los indicadores Niño $1+2$, Niño 3.4 y ONI tienen una frecuencia dominante del orden de 4 a 5 años. Los indicadores manchas solares, AMM, SOI, TSA y TNA tienen frecuencias dominantes entre 8.5 y 13 años. Mientras que los indicadores PDO y AMO presentan una frecuencia dominante multidecadal.

En el análisis de varianza que se indica en la Tabla 7 se observa que los procesos $N_{11}$ aportan un $66 \%$ a la varianza del espectro de las fluctuaciones. Mientras que los procesos $N_{5}$ tiene un importante aporte en los indicadores ONI, Niño 3.4 y Niño $1+2$.

Tabla 7. Aporte de la varianza en porcentaje a los distintos anchos de bandas analizados.

\begin{tabular}{|c|c|c|c|c|c|c|c|c|c|c|}
\hline & MS & AMO & AMM & ONI & PDO & $\begin{array}{l}\text { SO } \\
\mathbf{I}\end{array}$ & $\begin{array}{l}\text { Niño } \\
\mathbf{3 . 4}\end{array}$ & $\begin{array}{l}\text { Niño } \\
\mathbf{1}+\mathbf{2}\end{array}$ & TSA & TNA \\
\hline$N_{a}$ & $2 \%$ & $1 \%$ & $56 \%$ & $52 \%$ & $21 \%$ & $\begin{array}{c}44 \\
\%\end{array}$ & $49 \%$ & $64 \%$ & $43 \%$ & $52 \%$ \\
\hline$N_{5}$ & $4 \%$ & $0 \%$ & $0 \%$ & $40 \%$ & $19 \%$ & $\begin{array}{c}25 \\
\%\end{array}$ & $38 \%$ & $34 \%$ & $5 \%$ & $0 \%$ \\
\hline$N_{11}$ & $66 \%$ & $1 \%$ & $18 \%$ & $6 \%$ & $14 \%$ & $\begin{array}{c}18 \\
\%\end{array}$ & $6 \%$ & $0 \%$ & $12 \%$ & $14 \%$ \\
\hline$N_{\mathrm{bd}}$ & $5 \%$ & $10 \%$ & $1 \%$ & $0 \%$ & $9 \%$ & $2 \%$ & $0 \%$ & $0 \%$ & $7 \%$ & $5 \%$ \\
\hline$N_{v}$ & $20 \%$ & $86 \%$ & $3 \%$ & $0 \%$ & $29 \%$ & $0 \%$ & $0 \%$ & $0 \%$ & $25 \%$ & $21 \%$ \\
\hline
\end{tabular}




\section{Análisis de correlaciones entre las fluctuaciones de indicadores macroclimáticos y series de caudales medios anuales}

Conocer los indicadores macroclimáticos cuyas fluctuaciones puedan tener vinculación con las sequías en las distintas escalas de tiempo es una herramienta que ayuda a la predicción de tales fenómenos. En este apartado se analizan las correlaciones entre las fluctuaciones de indicadores macroclimáticos y de los caudales medios anuales, para distintos anchos de bandas. Las cuencas seleccionadas para este análisis son la de los ríos Suquía, Dulce, Paraná, San Juan y Atuel, por ser las series más extensas y representativas de cada región estudiada. Los filtros pasa-banda de Fourier utilizados fueron los siguientes: $N_{b d}$ : filtro pasa-banda entre 13 y 35 años; $N_{11}$ : filtro pasabanda entre 7-13 años; $N_{5}$ f filtro pasa-banda entre 3 y 7 años. En este análisis se calculó el coeficiente de correlación entre las series temporales de cada serie filtrada de caudales de cada cuenca $\left(N_{b d}\right.$, $N_{11}, N_{5}$ ) y las series filtradas de los índices macroclimáticos en el mismo ancho de banda. Luego se graficaron las series que poseen mejor correlación. A continuación se presentan los resultados para los ríos analizados.

\section{Río Dulce}

En la Tabla 8 se muestra el coeficiente de correlación entre las series de caudales del río Dulce y las series de los distintos indicadores.

Tabla 8. Coeficiente de correlación entre las series de caudales del río Dulce y las series de los distintos indicadores.

\begin{tabular}{|c|c|c|c|c|c|c|c|c|c|}
\hline \multicolumn{10}{|c|}{$\boldsymbol{N}_{\boldsymbol{b d}}$} \\
\hline TNA & MS & AMO & ONI & PDO & SOI & AMM & Niño 3.4 & Niño 1+2 & TSA \\
\hline 0.46 & 0.59 & 0.63 & 0.23 & 0.71 & -0.32 & 0.82 & 0.16 & 0.73 & -0.51 \\
\hline \multicolumn{10}{|c|}{$\mathbf{N}_{\mathbf{1 1}}$} \\
\hline
\end{tabular}




\begin{tabular}{|l|c|c|c|c|c|c|c|c|c|} 
TNA & MS & AMO & ONI & PDO & SOI & AMM & Niño 3.4 & Niño 1+2 & TSA \\
\hline-0.40 & -0.08 & 0.28 & -0.44 & -0.36 & 0.19 & -0.21 & -0.42 & 0.42 & -0.03 \\
\hline \multicolumn{10}{|c|}{$\mathbf{N}_{\mathbf{5}}$} \\
\hline TNA & MS & AMO & ONI & PDO & SOI & AMM & Niño 3.4 & Niño 1+2 & TSA \\
\hline 0.54 & 0.20 & -0.33 & 0.37 & 0.46 & -0.29 & 0.39 & 0.38 & 0.19 & 0.25 \\
\hline
\end{tabular}

A continuación, en las siguientes figuras, se muestran la evolución temporal de caudales y los indicadores macroclimáticos. Para graficar las series de caudales, éstas se normalizan restando la media y dividiendo por el desvío de cada serie.

En la Figura 17 se observa que las fases negativas del indicador AMM coincidieron con las sequías severas de la cuenca de mediados de la década de los años 40, 60 y fines de los 80 , y la sequía de los años 2011-2013. Se destaca la alta correlación encontrada entre la serie de caudales y los indicadores MS, AMO, PDO, Niño $1+2$ y TSA para el ancho de banda de 13 a 35 años. En la Figura 17b se observa que el indicador Niño $1+2$ en su fase negativa sucedió luego de dos años, por periodos de sequías en la cuenca. Este comportamiento se observa para las sequías de mediados de la década de los años 50 y 60 (sequías severas por su permanencia en el tiempo), y para las sequías de los años 1988-1989, 1993-1995, 2002-2004 y 2011-2013. En la Figura 17e se observa una correspondencia entre valles y picos para la mayoría de los ciclos observados, excepto para el periodo 1994-2004. En la Figura 18 se resalta que las fases positivas del TSA coinciden con la sequía de 1962-1971, y anteceden las sequías 19881990 y 2005-2010. Cabe señalar que desde 1950, estas sequías son las más largas que se identificaron a la fecha.

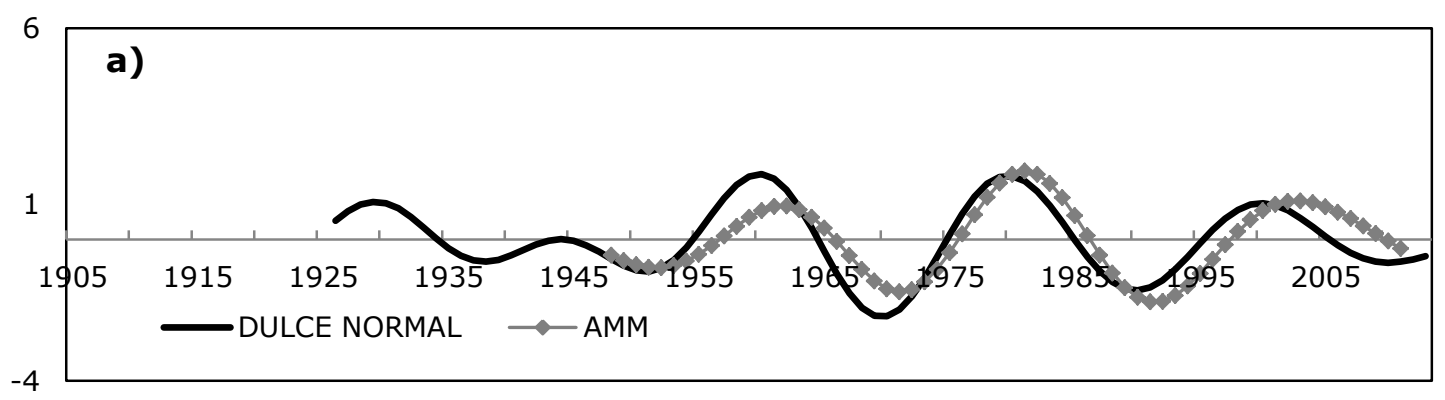



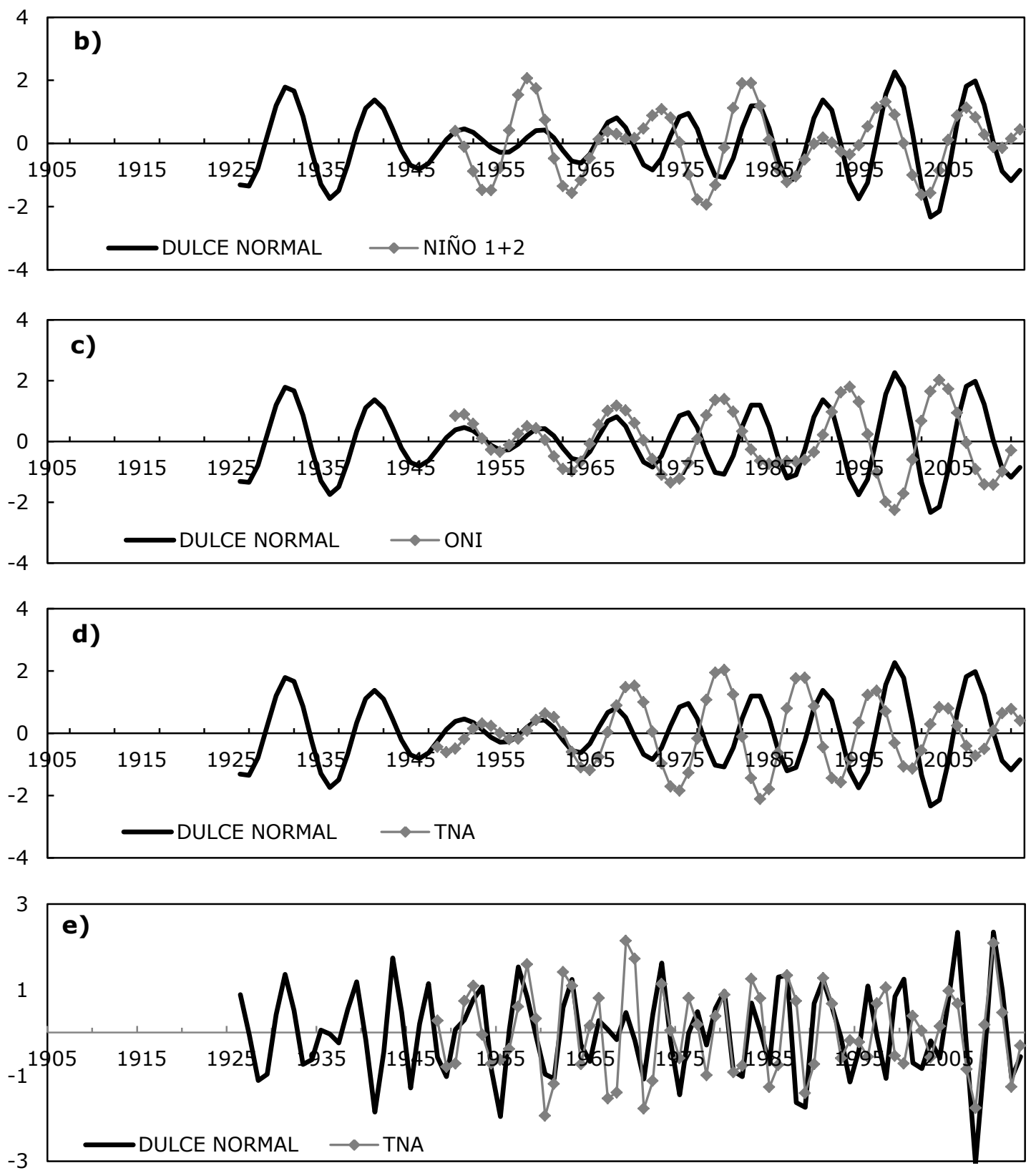

Figura 17. a) Serie de caudales normalizados del río Dulce y del indicador AMM ambas filtradas con filtro pasa-banda entre 13 y 35 años. Caudales normalizados del río Dulce con filtro pasa-banda entre 7 y 13 años, e indicadores Niño $1+2$ b) ONI c) y d) TNA; e) serie de caudales normalizados del río Dulce y del indicador TNA, ambas filtradas con filtro pasa-banda entre 3 y 7 años. 

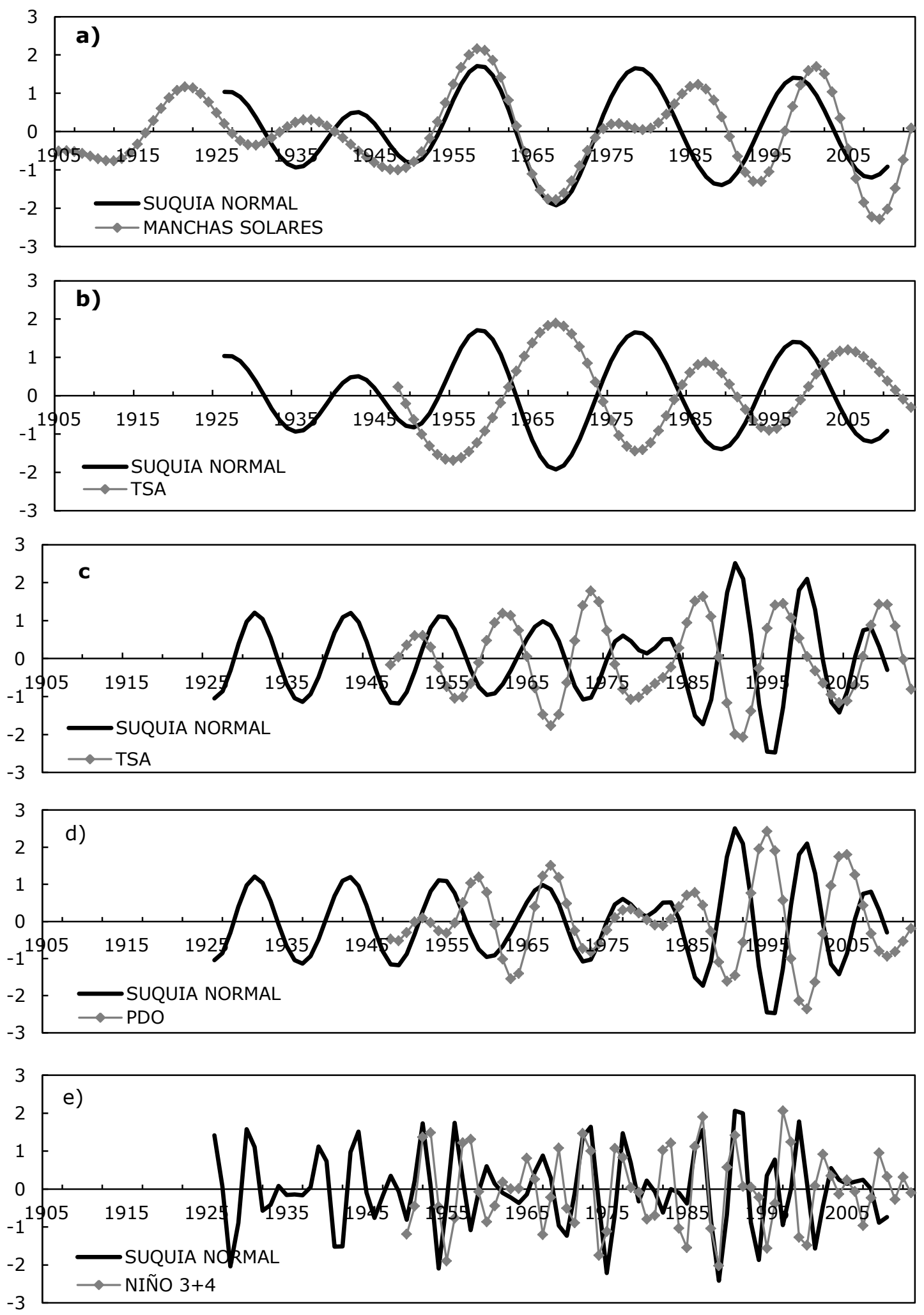

Figura 18. a) Serie de caudales del río Suquía y del indicador manchas solares; b) TSA, todos filtrados con filtro pasa-banda entre 13 y 35 años; c) caudales del río Suquía e indicador TSA; d) PDO, con filtro pasa-banda entre 7 y 13 años; e) serie 
de caudales del río Suquía y del indicador Niño 3.4, ambos filtrados con filtro pasabanda entre 3 y 7 años.

En la Figura 19a se observa que la fase positiva del indicador TSA coincide con déficits hídricos (sequía 1966-1972), y periodos de caudales bajos (no identificados como sequías hidrológicas) en los años 1985-1988 y 1999-2008. En la Figura 19c se observa que las fases negativas de indicador Niño $1+2$ coinciden con las sequías 1947-55 (en la etapa final), 1961-1963, 1977, 1995, y 2001-2005, y con la etapa de caudales bajos en 1986-1988 no identificadas como sequías hidrológicas. En la Figura 20a puede observarse que las fases negativas de indicador AMM concuerdan con los periodos de déficits hídricos en la cuenca, coincidiendo con las sequías identificadas en 1945-1952, 1966-1971, 1988-1996 y 2009-2013. Las fases negativas del indicador Niño 3.4 coinciden con los eventos de sequía de la cuenca en los años 1950-1951, 1960, 1962-1965, 1975, caudal bajo de 1985, 1988-1989, 1995-1996, 1999, 2004, 2007 (ver Figura 20c). En la Figura 21a se observa que las fases negativas del indicador AMM coinciden con los ciclos bidecadales de caudales bajos en la cuenca del río San Juan. Dentro de estas fases negativas se identificaron las sequías de $1945-53,1966-1975,1988-1996$ y 20102013. En la Figura 21d se observa que las fases negativas del Niño 3.4 coinciden con la mayoría de los eventos de sequía identificados. Estos eventos son 1954-1957, 1960-1962, 1964, 1974-1975, 19791981, 1985, 1988-1989, 1993-1996, 1999, 2007-2008.

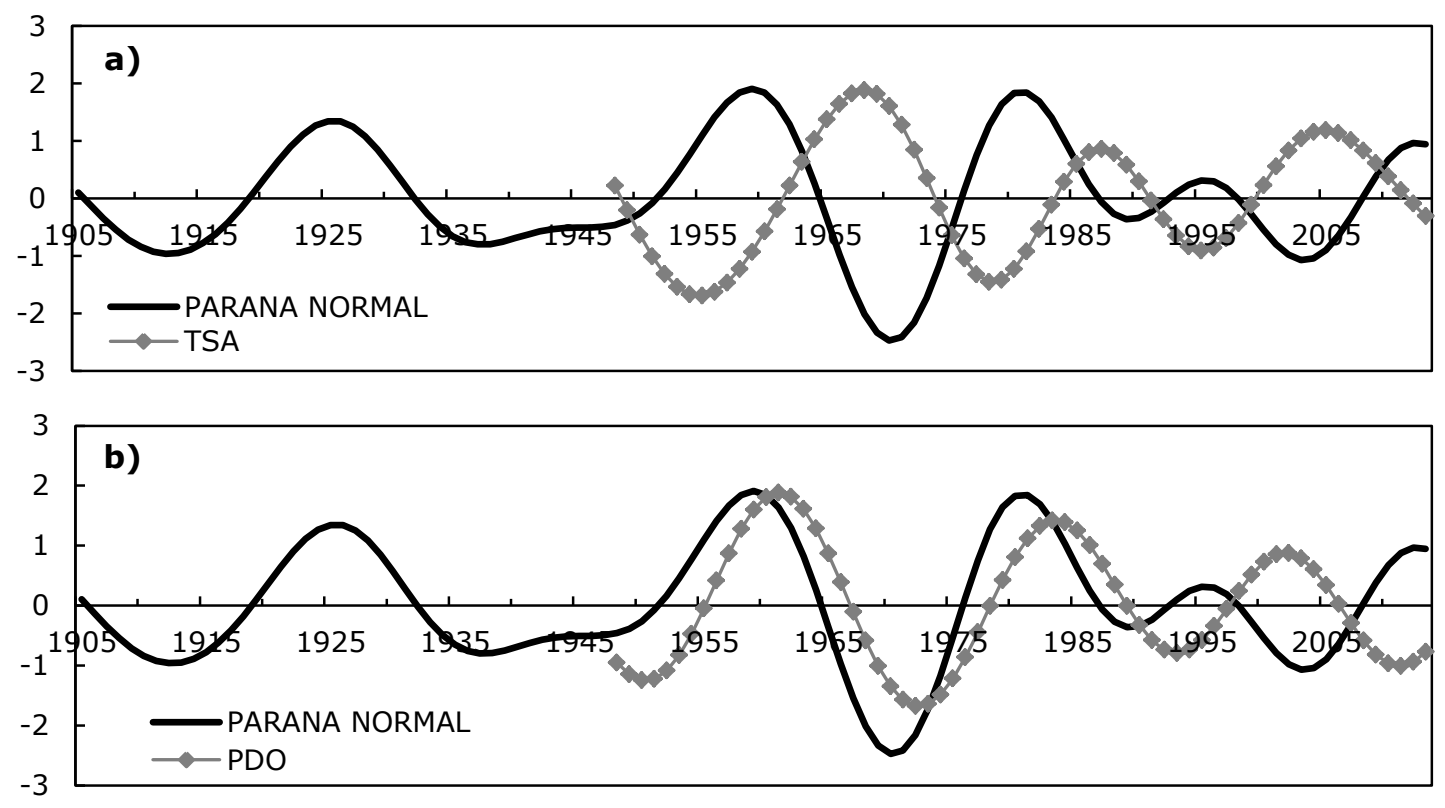



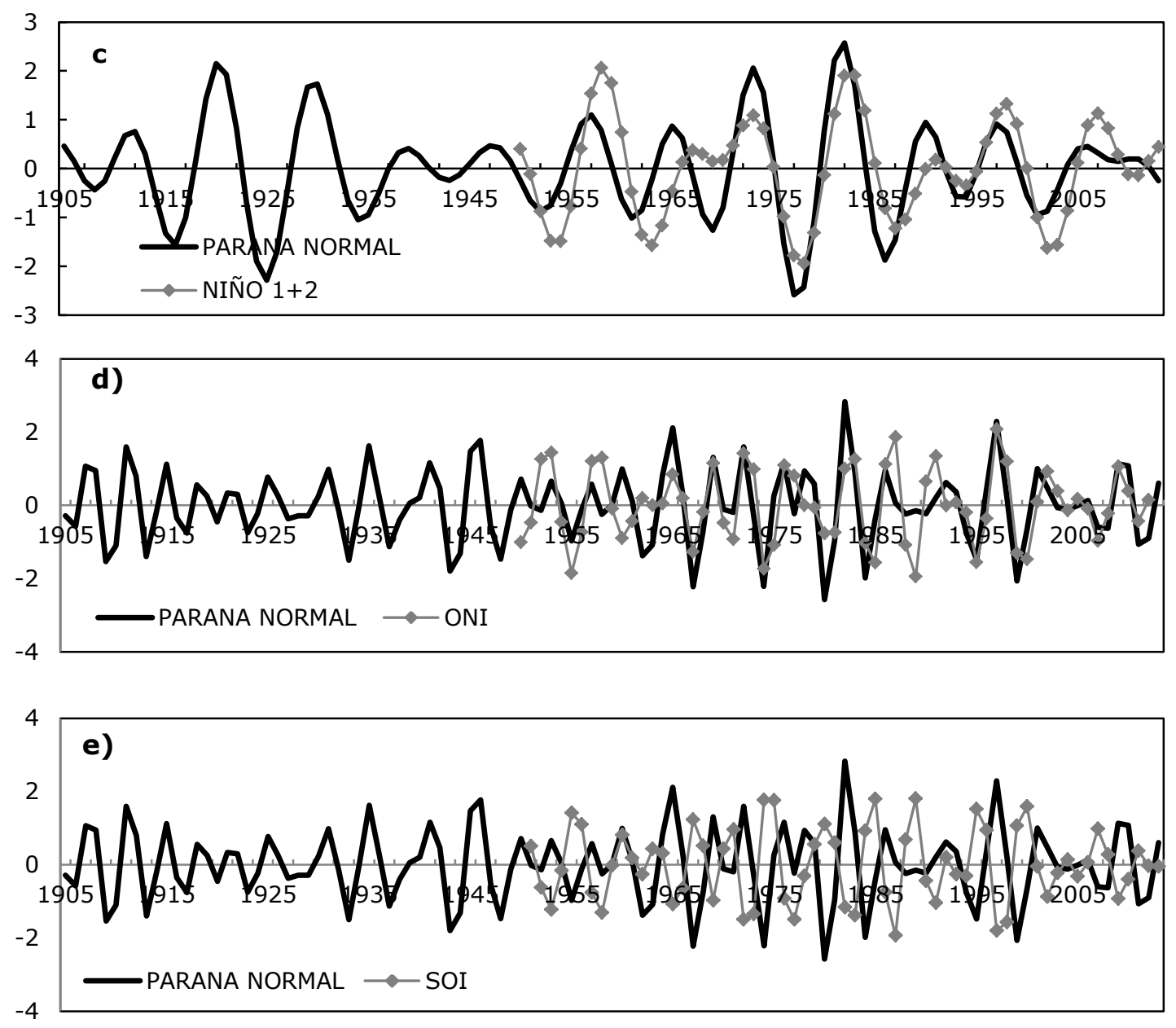

Figura 1. a) Serie de caudales del río Paraná y del indicador TSA; b) PDO, todos filtrados con filtro pasa-banda entre 13 y 35 años; c) serie de caudales del río Paraná y del indicador Niño 1+2, ambos filtrados con filtro pasa-banda entre 7 y 13 años; d) serie de caudales del río Paraná y del indicador ONI, y e) SOI, todos filtrados con filtro pasa-banda entre 3 y 7 años.

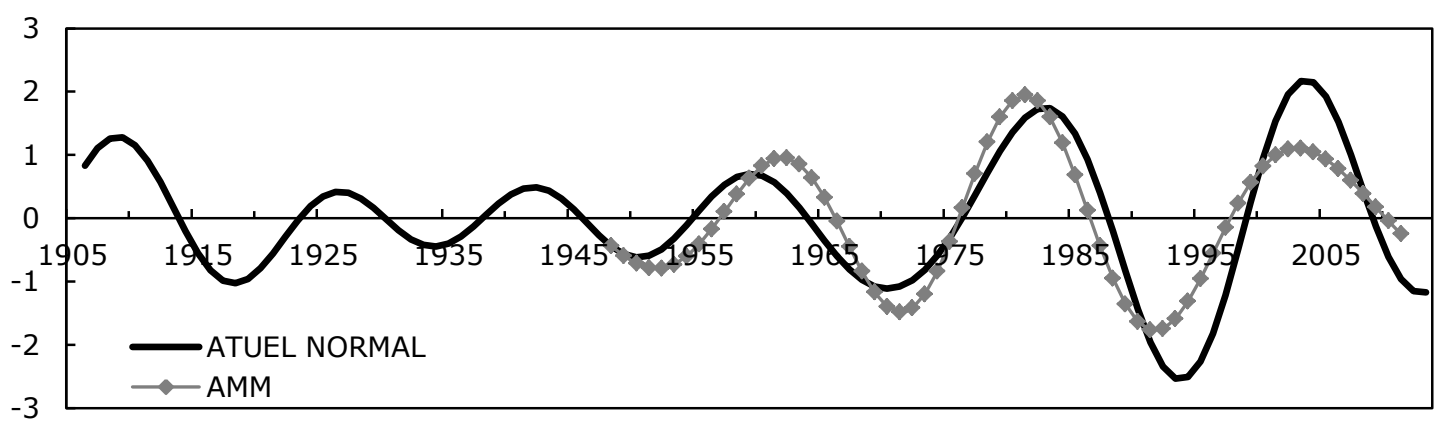



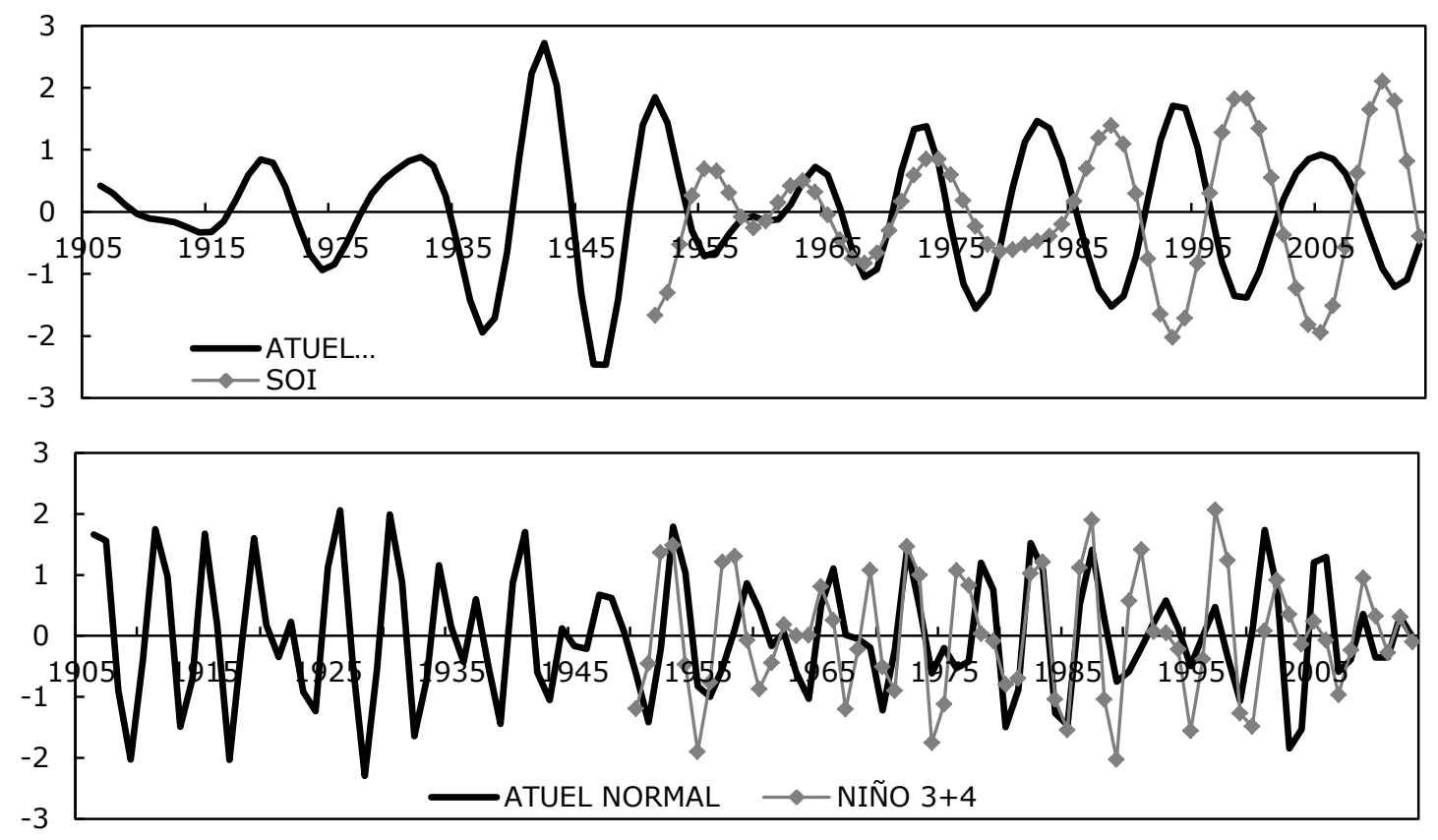

Figura 2. a) Serie de caudales del río Atuel y del indicador AMM, ambos filtrados con filtro pasa-banda entre 13 y 35 años; b) serie de caudales del río Atuel y del indicador SOI, ambos filtrados con filtro pasa-banda entre 7 y 13 años; c) serie de caudales del río Atuel y del indicador Niño 3.4, ambos filtrados con filtro pasabanda entre 3 y 7 años.

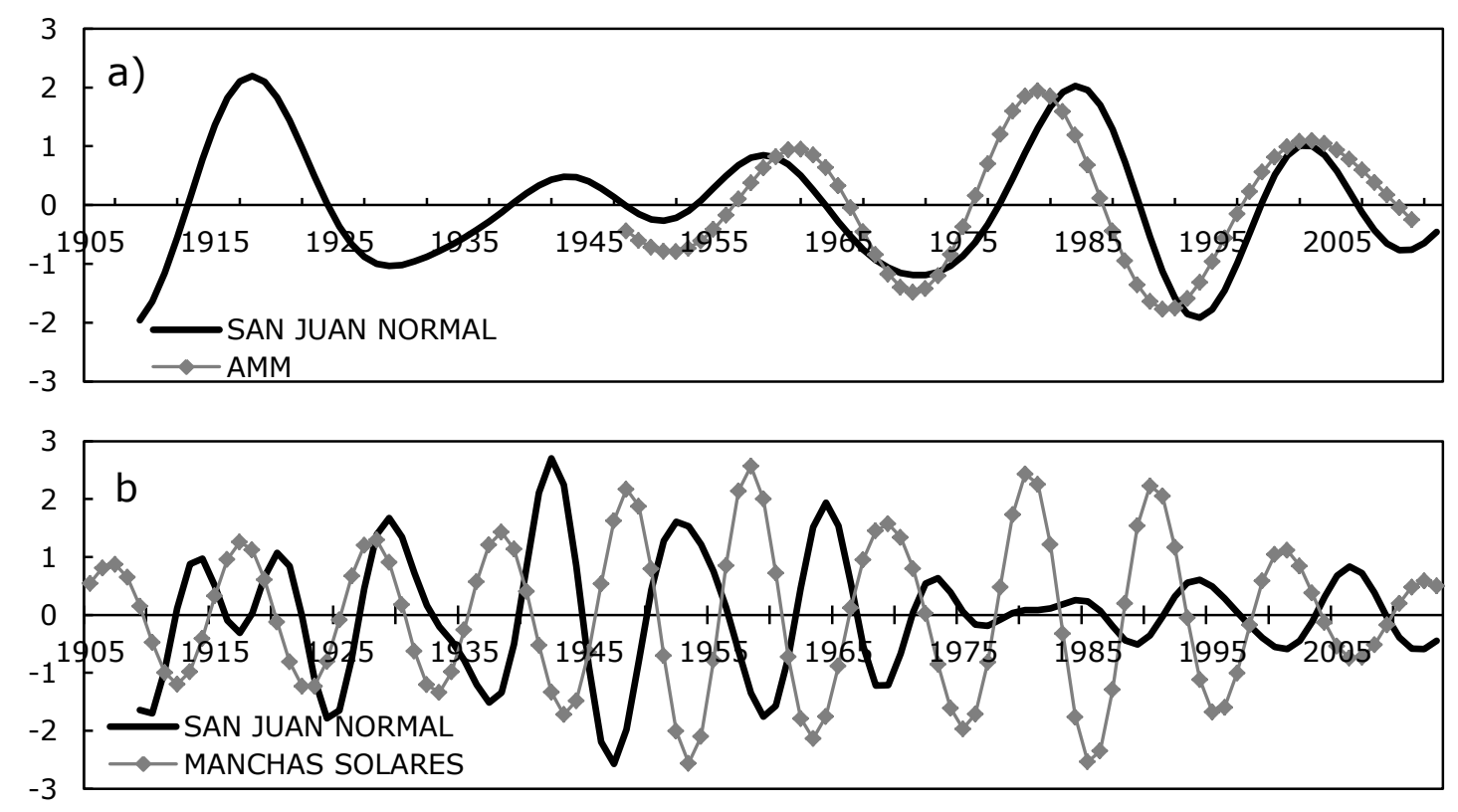



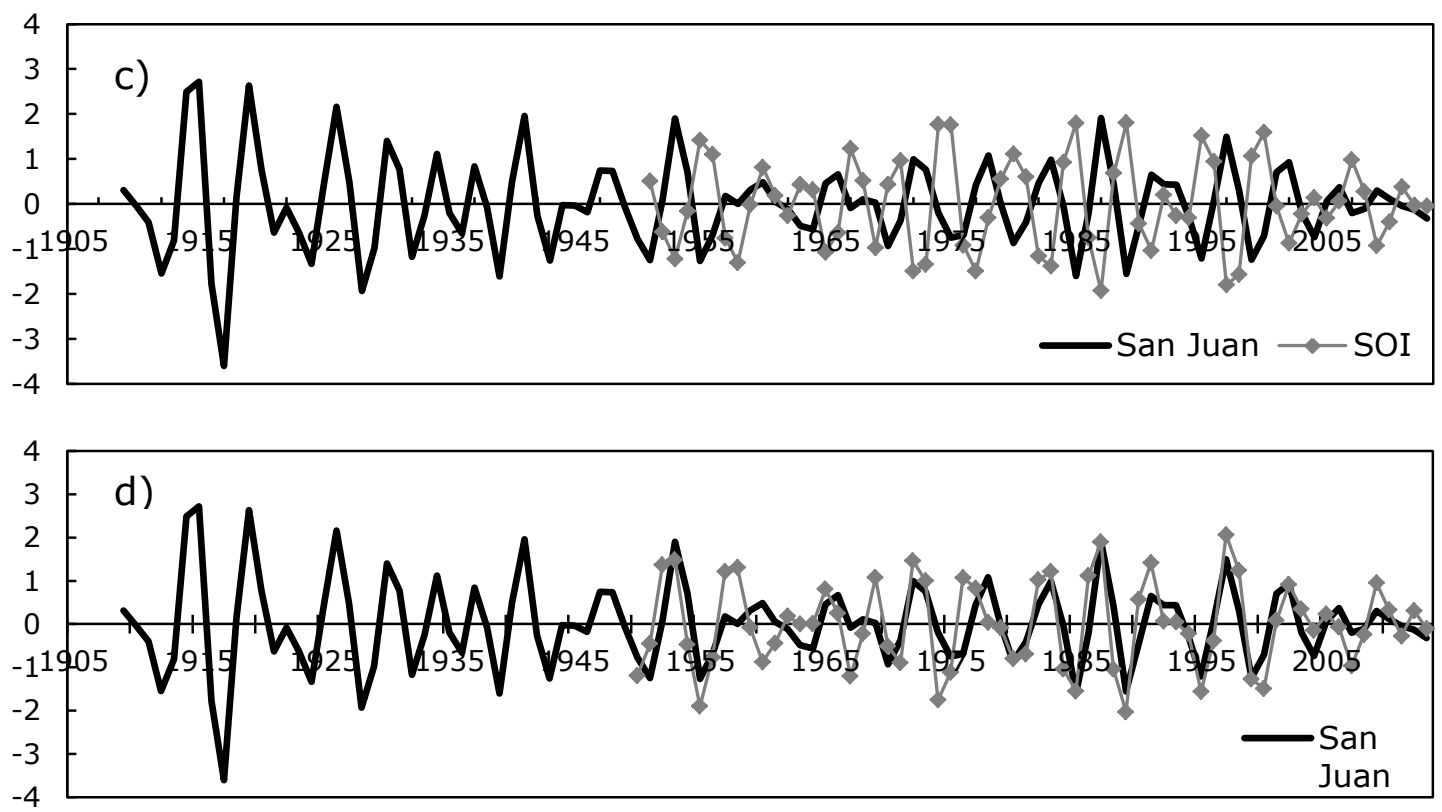

Figura 3. a) Serie de caudales del río San Juan y del indicador AMM, ambos filtrados con filtro pasa-banda entre 13 y 35 años; b) serie de caudales del río San Juan y del indicador manchas solares, ambos filtrados con filtro pasa-banda entre 7 y 13 años; c) serie de caudales del río San Juan y del indicador SOI, ambos filtrados con filtro pasa-banda entre 3 y 7 años; d) serie de caudales del río San Juan y del indicador Niño 3.4, ambos con filtro pasa-banda entre 3 y 7 años.

\section{Río Suquía}

En la Tabla 9 se observa el coeficiente de correlación entre las series de caudales del río Suquía y las series de los distintos indicadores.

Tabla 9. Coeficiente de correlación entre las series de caudales del río Suquía y las series de los distintos indicadores.

\begin{tabular}{|c|c|c|c|c|c|c|c|c|c|}
\hline \multicolumn{10}{|c|}{$\boldsymbol{N}_{\boldsymbol{b} d}$} \\
\hline TNA & MS & AMO & ONI & PDO & SOI & AMM & Niño 3.4 & Niño 1+2 & TSA \\
\hline 0.09 & 0.59 & 0.25 & -0.01 & 0.39 & -0.15 & 0.51 & -0.16 & 0.58 & -0.74 \\
\hline \multicolumn{10}{|c|}{ N11 } \\
\hline TNA & MS & AMO & ONI & PDO & SOI & AMM & Niño 3.4 & Niño 1+2 & TSA \\
\hline-0.21 & 0.11 & 0.28 & -0.15 & -0.51 & 0.00 & 0.01 & -0.13 & 0.01 & -0.53 \\
\hline \multicolumn{10}{|c|}{ N5 } \\
\hline TNA & MS & AMO & ONI & PDO & SOI & AMM & Niño 3.4 & Niño 1+2 & TSA \\
\hline
\end{tabular}




\begin{tabular}{|l|l|l|l|l|l|l|l|l|l|}
\hline 0.03 & -0.09 & -0.04 & 0.20 & 0.05 & -0.15 & -0.08 & 0.22 & 0.12 & 0.19 \\
\hline
\end{tabular}

\section{Río Paraná}

En la Tabla 10 se tiene el coeficiente de correlación entre las series de caudales del río Paraná y las series de los distintos indicadores.

Tabla 10. Coeficiente de correlación entre las series de caudales del río Paraná y las series de los distintos indicadores.

\begin{tabular}{|c|c|c|c|c|c|c|c|c|c|}
\hline \multicolumn{1}{|c|}{$\boldsymbol{N}_{\boldsymbol{b d}}$} \\
\hline TNA & MS & AMO & ONI & PDO & SOI & AMM & Niño 3.4 & Niño 1+2 & TSA \\
\hline 0.36 & 0.45 & 0.39 & 0.36 & 0.54 & -0.53 & 0.53 & 0.13 & 0.27 & -0.69 \\
\hline \multicolumn{10}{|c|}{$\boldsymbol{N}_{\mathbf{1 1}}$} \\
\hline TNA & MS & AMO & ONI & PDO & SOI & AMM & Niño 3.4 & Niño 1+2 & TSA \\
\hline-0.06 & 0.17 & 0.11 & -0.13 & -0.19 & 0.13 & -0.09 & -0.06 & 0.68 & 0.07 \\
\hline \multicolumn{10}{|c|}{$\boldsymbol{N}_{\mathbf{5}}$} \\
\hline TNA & MS & AMO & ONI & PDO & SOI & AMM & Niño 3.4 & Niño 1+2 & TSA \\
\hline 0.07 & -0.28 & -0.03 & 0.60 & 0.34 & -0.59 & -0.07 & 0.58 & 0.57 & -0.34 \\
\hline
\end{tabular}

En la Figura 19 se presentan los más significativos.

\section{Río Atuel}

En la Tabla 11 se observa el coeficiente de correlación entre las series de caudales del río Atuel y las series de los distintos indicadores.

Tabla 11. Coeficiente de correlación entre las series de caudales del río Atuel y las series de los distintos indicadores.

\begin{tabular}{|c|c|c|c|c|c|c|c|c|c|}
\hline \multicolumn{10}{|c|}{$\boldsymbol{N}_{\boldsymbol{b d}}$} \\
\hline TNA & SOL & AMO & ONI & PDO & SOI & AMM & Niño 3.4 & Niño 1+2 & TSA \\
\hline 0.62 & 0.37 & 0.64 & 0.02 & 0.65 & -0.02 & 0.87 & 0.08 & 0.42 & 0.06 \\
\hline \multicolumn{10}{|c|}{$N_{11}$} \\
\hline
\end{tabular}


Ciencias $₫$ Agua

\begin{tabular}{|c|c|c|c|c|c|c|c|c|c|} 
TNA & SOL & AMO & ONI & PDO & SOI & AMM & Niño 3.4 & Niño 1+2 & TSA \\
\hline-0.34 & -0.27 & 0.24 & 0.43 & 0.22 & -0.62 & -0.41 & 0.41 & 0.22 & 0.00 \\
\hline \multicolumn{10}{|c|}{$\mathbf{N}_{\mathbf{5}}$} \\
\hline TNA & SOL & AMO & ONI & PDO & SOI & AMM & Niño 3.4 & Niño 1+2 & TSA \\
\hline 0.05 & -0.11 & 0.11 & 0.45 & 0.26 & -0.50 & -0.14 & 0.46 & 0.38 & -0.14 \\
\hline
\end{tabular}

En la Figura 20 y Figura 21 se presentan los más significativos.

\section{Río San Juan}

En la Tabla 12 se muestra el coeficiente de correlación entre las series de caudales del río San Juan y las series de los distintos indicadores.

Tabla 12. Coeficiente de correlación entre las series de caudales del río San Juan y las series de los distintos indicadores.

\begin{tabular}{|c|c|c|c|c|c|c|c|c|c|}
\hline \multicolumn{10}{|c|}{$\boldsymbol{N}_{\boldsymbol{b d}}$} \\
\hline TNA & MS & AMO & ONI & PDO & SOI & AMM & Niño 3+4 & Niño 1+2 & TSA \\
\hline 0.39 & 0.49 & 0.30 & 0.19 & 0.63 & -0.29 & 0.81 & 0.14 & 0.42 & -0.15 \\
\hline \multicolumn{8}{|c|}{$\mathbf{N}_{\mathbf{1 1}}$} \\
\hline TNA & MS & AMO & ONI & PDO & SOI & AMM & Niño 3+4 & Niño 1+2 & TSA \\
\hline-0.27 & -0.46 & 0.36 & 0.04 & -0.07 & -0.22 & -0.32 & -0.05 & -0.30 & 0,20 \\
\hline \multicolumn{8}{|c|}{$\boldsymbol{N}_{\mathbf{5}}$} \\
\hline TNA & MS & AMO & ONI & PDO & SOI & AMM & Niño 3+4 & Niño 1+2 & TSA \\
\hline 0.23 & -0.23 & 0.07 & 0.69 & 0.60 & -0.72 & -0.16 & 0.70 & 0.62 & 0.05 \\
\hline
\end{tabular}

\section{Conclusiones}


En este trabajo se identificaron las periodicidades plurianuales en series de caudales escurridos en sistemas fluviales de la República Argentina. El análisis espectral realizado permite determinar que para $86 \%$ de las series de caudales analizadas, la componente aleatoria (alta frecuencia) explica entre 30 y $50 \%$ de las señales de caudales. Además se observó que existe una variabilidad en las series de alta, baja y media frecuencia que aportan a la fluctuación de los caudales en distintos porcentajes. Luego de la componente aleatoria, los procesos con predominio son los que tienen periodos dominantes entre 3 y 7 años ( $p$. ej., ENSO), y los que tienen periodos entre 13 y 35 (oscilaciones bidecadales). Los resultados obtenidos en este trabajo permiten avanzar en el estudio de los procesos que se deben considerar en la explicación de fenómenos hidrológicos, como sequías y exceso de agua.

A nivel bidecadal se encontró una gran influencia del océano Atlántico sobre las series de caudales. En las cinco cuencas analizadas (ríos Suquía, Dulce, Paraná, Atuel y San Juan) se observó una alta correlación con el índice Atlantic Meridional Mode "AMM", Dulce $(0,82)$; Paraná $(0,53)$; Atuel $(0,87)$; San Juan $(0,81)$ y Suquía $(0,51)$. Siendo la más alta correlación encontrada en casi todas las cuencas, excepto para el Paraná y Suquía, que presentan una alta correlación negativa con el TSA Paraná $(-0.69)$ y Suquía $(-0.74)$. A nivel decadal se observan correlaciones significativas (mayores a 0.5) para el Paraná con el Niño $1+2(0.68)$, el Atuel con el SOI $(-0.62)$ y Suquía con TSA (-0.53).

En la escala plurianual se observan correlaciones negativas con el SOI, para las cuencas de los ríos Paraná $(-0.59)$, Atuel $(-0.50)$ y San Juan (-0.72). Fuertes correlaciones positivas con los indicadores ONI para el Paraná (0.60); San Juan y Atuel, con el Niño 3.4 (0.70; 0.46 respectivamente), y el Dulce con el TNA (0.54).

Esto no sólo confirma los resultados de trabajos anteriores (Compagnucci et al., 2014; Mauas, Buccino y Flamenco, 2011; Dölling, 2014; Vargas et al., 2002), además aporta nueva información con respecto a vínculos con indicadores del océano Atlántico para las distintas cuencas. Estos resultados son importantes para el entendimiento de la dinámica macroclimática de Argentina y de su pronóstico en el espacio y en el tiempo.

\section{Agradecimientos}


Al Consejo Nacional de Investigaciones Científicas y Técnicas de Argentina (Conicet) por la beca otorgada para desarrollar este trabajo.

\section{Referencias}

Compagnucci, R. H., Berman, A. L., Velasco-Herrera, V., \& Silvestri, G. (2014). Are southern South American Rivers linked to the solar variability? International Journal of Climatology, 34(5), 1706-1714.

Correa, N. R., \& Guevara, P. E. (1992). Periodicidades plurianuales de una serie de escurrimientos. Revista Ingeniería UC, 1(1), 8-18.

Díaz, E., Rodríguez, A., Dölling, O., Bertoni, J. C., \& Smrekar, M. (2016). Identificación y caracterización de sequías hidrológicas en Argentina. Tecnología y Ciencias del Agua, 7(1), 125-133.

Dölling, O. (2014). Tendencias en el escurrimiento de los ríos argentinos a partir del análisis de las manchas solares y de la temperatura del Pacífico. Revista Hydria, 51, 22.

Fernández, B. (1997). Identificación y caracterización de sequías hidrológicas en Chile Central. Ingeniería del Agua, 4(4), 37-46.

Mauas, P. J., Buccino, A. P., \& Flamenco, E. (2011). Long-term solar activity influences on South American rivers. Journal of Atmospheric and Solar-Terrestrial Physics, 73(2), 377-382.

Paoli, C., \& Schreider, M. (2000). El río Paraná en su tramo medio. Una contribución al conocimiento y prácticas ingenieriles en un gran río de llanura. Santa Fé, Argentina: Centro de publicaciones de la Universidad Nacional del Litoral.

Vargas, W. M., Minetti, J. L., \& Poblete, A. G. (2002). Low-frequency oscillations in climatic and hydrological variables in southern South America's tropical-subtropical regions. Theoretical and applied climatology, 72(1-2), 29-40.

NOAA, National Oceanic \& Atmospheric Administration. (2016). Climate Indices: Monthly Atmospheric and Ocean Time Series. Recuperado de http://www.esrl.noaa.gov/psd/data/climateindices/list/

SILSO, Sunspot Index and Long-Term Solar Observations. (2016). Sunspot Number. Recuperado de http://www.sidc.be/silso/datafiles 
Subsecretaría de Recursos Hídricos. (2010). Cuencas y regiones hídricas superficiales de la RA (CD). Buenos Aires, Argentina: Subsecretaría de Recursos Hídricos. 